\title{
Q-Curvature, Spectral Invariants, and Representation Theory ${ }^{\star}$
}

\author{
Thomas P. BRANSON ${ }^{\dagger}$ \\ $\dagger$ Deceased \\ URL: http://www. math.uiowa.edu/ branson/
}

Received August 01, 2007 from Xingwang Xu; Published online September 16, 2007

Original article is available at http://www.emis.de/journals/SIGMA/2007/090/

\begin{abstract}
We give an introductory account of functional determinants of elliptic operators on manifolds and Polyakov-type formulas for their infinitesimal and finite conformal variations. We relate this to extremal problems and to the $Q$-curvature on even-dimensional conformal manifolds. The exposition is self-contained, in the sense of giving references sufficient to allow the reader to work through all details.
\end{abstract}

Key words: conformal differential geometry; functional determinant; conformal index

2000 Mathematics Subject Classification: 58J52; 53A30

During May and June in 2004, the Institute of Mathematical Sciences, at the National University of Singapore, held a program on geometric partial differential equations. The program had invited several instructional lecturers. Professor Thomas Branson was one of them. Originally we had planned to publish the lecture notes of the instructional lecturers. This paper was submitted by Professor Branson for that purpose. In fact this was the only paper we had received by the deadline. Hence we decided to cancel the plan for a proceedings volume. Just after that period, Thomas Branson unexpectedly passed away. We held the paper without knowing what we could do with it. When the editors of this proceedings volume invited me to submit an article, I realised that this would be an ideal place for Professor Thomas Branson's paper. I immediately submitted the paper to editors of the current proceedings. I would like to take this opportunity to express my sincere appreciation to the editors for their help.

\section{Xingwang Xu (National University of Singapore)}

E-mail: matxuxw@nus.edu.sg

\section{The functional determinant}

In order to get a feel for the spectral theory of natural differential operators on compact manifolds, recall the idea of Fourier series, where one attempts to expand complex functions on the unit circle $\mathbf{S}^{1}$ in $\mathbb{C}$ in the form

$$
\sum_{k=-\infty}^{\infty} c_{k} e^{\mathrm{i} k \theta}=c_{0}+\sum_{j=1}^{\infty}\left(a_{j} \cos j \theta+b_{j} \sin j \theta\right) .
$$

The trigonometric series is an expansion in real eigenfunctions of the Laplacian $\Delta=-d^{2} / d \theta^{2}$ (the eigenvalue being $j^{2}$ ). The exponential series is an expansion in eigenfunctions of the operator $-\mathrm{i} d / d \theta$, which is a square root of the Laplacian; the eigenvalue is $k$.

\footnotetext{
${ }^{\star}$ This paper is a contribution to the Proceedings of the 2007 Midwest Geometry Conference in honor of Thomas P. Branson. The full collection is available at http://www.emis.de/journals/SIGMA/MGC2007.html
} 
Suppose we wish to find the determinant of the Laplacian on the circle, or of its square root. Taking the naive view that the determinant should be the product of the eigenvalues in some spectral resolution, we immediately have some problems - we are really being asked to take the product of the nonnegative integers. We could declare this to be 0 by convention ( 0 being one of the eigenvalues), but the question of computing a determinant like

$$
\operatorname{det}\left(-\frac{d^{2}}{d \theta^{2}}+1\right)
$$

still persists - is there any way to make sense of such determinants?

There are, of course, other characterizations of the determinant of a finite-dimensional linear transformation $T$, and we might try to generalize one of these. For example, there is the exterior algebra definition, which appears in abstract index notation (see below for an explanation) as

$$
\operatorname{det} T=T_{\left[a_{1}\right.}^{a_{1}} T_{a_{2}}^{a_{2}} \cdots T_{\left.a_{m}\right]}^{a_{m}} .
$$

There is also a zeta function definition: let

$$
\zeta_{T}(s)=\operatorname{Tr} T^{-s}, \quad s \in \mathbb{C} .
$$

(The exponential functions $z \mapsto z^{-s}$ must be well-defined on $\operatorname{spec}(T)$; for this, $\operatorname{since} \operatorname{spec}(T)$ is finite, we just need $0 \notin \operatorname{spec}(T)$.) The determinant is then

$$
\operatorname{det} T=e^{-\zeta_{T}^{\prime}(0)} .
$$

Indeed, (1) follows from the calculation

$$
\left.\frac{d}{d s}\right|_{s=0} \operatorname{Tr} T^{-s}=-\operatorname{Tr} \log T
$$

The particular branch cut used to compute $\log T$ affects the value of $\zeta_{T}^{\prime}(0)$ (moving it by multiples of $2 \pi \mathrm{i}$ ), but has no effect on its exponential.

In fact, the zeta function approach is a fruitful one for operators like the Laplacian on $\mathbf{S}^{1}$. Before pursuing it more deeply, however, let us look at still another approach. If $T$ is symmetric and does not have 0 in its spectrum, then

$$
\operatorname{det} T=\pi^{m}\left(\int_{\mathbb{R}^{m}} e^{-(T x, x)} d x\right)^{-2}
$$

This is, in fact, the sort of formula that one tries to imitate in quantum theory, with the so-called functional integrals - integrals over function space. Since there are problems assigning meaning to such integrals, one tries to evaluate the infinite-dimensional analogue of the left side of (2) instead. Among the possible interpretations (or regularizations) of the determinant, one may choose the zeta function, and if the operator in question has certain properties, this succeeds in assigning a value.

To see what zeta function regularization says about $\operatorname{det}(\Delta)$ on $\mathbf{S}^{1}$, let us just brutally remove the 0 eigenvalue. This makes the zeta function

$$
\zeta_{\Delta}(s)=\sum_{j=1}^{\infty} 2 j^{-2 s}=2 \zeta_{\mathrm{Riem}}(2 s),
$$

where $\zeta_{\text {Riem }}$ is the Riemann zeta function. As a result,

$$
\operatorname{det} \Delta=e^{-4 \zeta_{\text {Riem }}^{\prime}(0)}=(2 \pi)^{2} .
$$


If we could take the eigenvalue product interpretation of the determinant literally, this would say that the product of the positive integers is $\sqrt{2 \pi}$.

Let us recall the process which assigns meaning to the expression $\zeta_{\mathrm{Riem}}^{\prime}(0)$. The series $\sum_{j=1}^{\infty} j^{-s}$ converges uniformly and absolutely on the half-plane for $\operatorname{Re}(s)>1$. The resulting holomorphic function has an analytic continuation which may be constructed using Dirichlet series and the Mellin transform, to a meromorphic function on $\mathbb{C}$, with a single simple pole at $s=1$.

What we would like to explore here is a generalization of this thinking to a situation in which:

- $\mathbf{S}^{1}$ is replaced by a compact $n$-dimensional smooth manifold;

- the Laplacian is replaced by a differential operator with positive definite leading symbol.

Roughly speaking, the same construction will go through in this setting, and there will be a functional determinant.

For example, consider the Laplacian of an $n$-dimensional Riemannian manifold $\left(M^{n}, g\right)$. One has the Riemannian connection $\nabla$, which allows covariant differentiation of vector fields, and in fact of all kinds of tensors. If $f$ is a smooth function, then $\nabla \nabla f$ is a 2-tensor. Contracting, one gets

$$
\Delta f:=-g^{a b} \nabla_{a} \nabla_{b} f=-\nabla^{a} \nabla_{a} f
$$

in abstract index notation. Here indices which are repeated, once up and once down, denote contractions. Indices are raised and lowered using the metric, and the number and position of indices just indicate a tensor's rank and covariance type (rather than any choice of local frame). For more on abstract index notation, see [31].

The leading symbol of a differential operator $D$ is, more or less, what one gets by writing $D$ in local coordinates (and frames, if $D$ is vector bundle valued), replacing each $\partial / \partial x^{a}$ by an i $\xi_{a}$, and keeping only the terms with the maximal number of $\xi$ 's. Here $\xi$ is an indeterminate covector field, or one-form; the result is some sort of function on the cotangent bundle of $M$. A precise formula for the leading symbol may be given, without making choices of local frames, as follows. Suppose $D$ has order $k$, and carries sections of a vector bundle $\mathbb{V}$ to sections of a vector bundle $\mathbb{W}$. Then if $f$ is a smooth function and $\varphi$ is a smooth section of $\mathbb{V}$,

$$
\sigma_{k}(D)\left(x,(d f)_{x}\right) \varphi=\lim _{t \rightarrow \infty} \frac{\left(D\left(e^{\mathrm{i} t f} \varphi\right)\right)_{x}}{t^{k}}
$$

where the subscript $x$ denotes the value of a section (in this case, of the cotangent bundle or $\mathbb{W}$ ) at $x$. Since any covector $\xi$ at $x$ is $(d f)_{x}$ for some $f$, this completely (and, as is easily checked, consistently) determines $\sigma_{k}(D)(x, \xi) \varphi$.

For example, in the case of the Laplacian on ordinary functions,

$$
\Delta f=-g^{a b} \partial_{a} \partial_{b}+(\text { lower order })
$$

so the leading symbol is

$$
\sigma_{2}(\Delta)(x, \xi)=g^{a b}(x) \xi_{a} \xi_{b} .
$$

The "2" in the $\sigma_{2}$ notation just keeps track of the order of the operator (the maximal homogeneity in $\xi$ ). In this sense, the leading symbol of the Laplacian "is" the metric (or, more precisely, the metric inverse $\left.g^{a b}\right)$.

Back in the general situation, $\sigma_{k}(D)$ may be thought of as a section of the vector bundle

$$
\operatorname{Hom}\left(\operatorname{Symm}\left(T^{*} M \otimes \cdots \otimes T^{*} M\right) \otimes \mathbb{V}, \mathbb{W}\right),
$$


using the identification of $k$-homogeneous functions on a vector space (here, the cotangent space $\left.T_{x}^{*} M\right)$ with symmetric $k$-tensors on that space. The construction of a total symbol is another matter. One may view this as depending on choices (i.e., as a function carrying atlases of $M$ which locally frame $\mathbb{V}$ and $\mathbb{W}$ to local total symbols) as in [23]. Or, one can assume more geometric structure and use it to try to define total symbols invariantly, as in [38]. This will not be an explicit issue here, though it is an issue in the underlying analysis of pseudo-differential operators, which provides the heat operators and complex powers of elliptic operators on which zeta function regularization relies. Roughly speaking, a total symbol will keep track of what happens to the operator under local Fourier transforms.

The Laplacian's leading symbol has a precise positivity property: if $\xi \neq 0$, then

$$
g^{a b} \xi_{a} \xi_{b}>0
$$

by the positive definiteness of the Riemannian metric. We can speak of something like this in the vector bundle valued case, say for a $k^{\text {th }}$ order differential operator carrying sections of a vector bundle $\mathbb{V}$ to sections of the same bundle $\mathbb{V}$, replacing positivity by positive definiteness, at least if $\mathbb{V}$ has a positive definite metric. We say that $D$ has positive definite leading symbol if for each $\xi \neq 0$ in $T_{x}^{*} M$, for each $x \in M$, we have

$$
\sigma_{k}(D)(x, \xi) \text { positive definite in } \operatorname{End}\left(\mathbb{V}_{x}\right) \text {. }
$$

A positive definite endomorphism $A$ is, of course, one with $(A v, v)>0$ for each nonzero vector $v$, where $(\cdot, \cdot)$ is the inner product (provided here by the metric on the bundle $\mathbb{V}$ ).

Note that only even-order operators can have positive definite leading symbol, since if $k$ is odd and $\sigma_{k}(D)(x, \xi)$ is positive definite, then $\sigma_{k}(D)(x,-\xi)$ is negative definite.

Perhaps the easiest examples of operators with positive definite leading symbol which do not have the form

$$
\Delta^{h}+\text { (lower order) }
$$

are the differential form operators $\delta d+a d \delta$, where $d$ and $\delta$ are the exterior derivative and coderivative, and $a$ is a positive constant not equal to 1 . To be more specific, if $\varphi$ is a $p$-form, then $d \varphi$ is the $(p+1)$-form

$$
(d \varphi)_{a_{0} \ldots a_{p}}=(p+1) \nabla_{\left[a_{0}\right.} \varphi_{\left.a_{0} \ldots \hat{a}_{s} \ldots a_{p}\right]},
$$

and $\delta \varphi$ is the $(p-1)$-form

$$
(\delta \varphi)_{a_{2} \ldots a_{p}}=-\nabla^{b} \varphi_{b a_{2} \ldots a_{p}} .
$$

In fact, one does not need a connection to define $d$; the right side of (3) is invariant as $\nabla$ ranges over all symmetric affine connections. $\delta$ does depend on the connection $\nabla$, and in fact is the formal adjoint of $d$ in the form metrics

$$
(\varphi, \psi)_{p}=\frac{1}{p !} \varphi^{a_{1} \ldots a_{p}} \psi_{a_{1} \ldots a_{p}} .
$$

This means that if $\varphi$ is a $p$-form and $\eta$ is a $(p+1)$-form on a Riemannian manifold $(M, g)$, then

$$
\int_{M}(d \varphi, \eta)_{p+1} d v_{g}=\int_{M}(\varphi, \delta \eta)_{p} d v_{g}
$$

provided either $\varphi$ or $\eta$ has compact support. Here $d v_{g}$ is the Riemannian measure. The formal adjoint property makes it clear that $\delta$ (unlike $d$ ) will generally change if we vary the metric $g$. 
The form Laplacian

$$
\Delta_{\text {form }}=\delta d+d \delta
$$

is related to the Laplacian $\nabla^{*} \nabla$ (sometimes called the Bochner Laplacian in this context) by the Weitzenböck formula

$$
\delta d+d \delta=\nabla^{*} \nabla+\mathcal{W} .
$$

Here $\mathcal{W}$, the Weitzenböck operator, is an action of the curvature tensor which has order 0 as a differential operator. As a result,

$$
\sigma_{2}\left(\Delta_{\text {form }}\right)(x, \xi)=|\xi|^{2} \operatorname{Id}_{\text {form }},
$$

where the length is measured by the metric: $|\xi|^{2}=g^{a b} \xi_{a} \xi_{b}$.

But if $D$ has order $k$ and $E$ has order $\ell$, then

$$
\sigma_{k+\ell}(D E)(x, \xi)=\sigma_{k}(D)(x, \xi) \sigma_{\ell}(E)(x, \xi) .
$$

In addition, formal adjoints (denoted by the superscript $*$ ) exist and are locally determined, and

$$
\sigma_{k}(D)(x, \xi)^{*}=\sigma_{k}\left(D^{*}\right)(x, \xi) .
$$

Thus $\delta d+a d \delta$ has positive semidefinite leading symbol for $a \geq 0$, and for $a>0$, the calculation

$$
(\delta d+a d \delta)\left(\delta d+a^{-1} d \delta\right)=\Delta_{\text {form }}^{2}
$$

(based on $d d=0$ ) shows that $\delta d+a d \delta$ has invertible leading symbol. Thus $\delta d+a d \delta$ has positive definite leading symbol for $a>0$.

Beyond differential forms, in other natural bundles, the idea of positive definite leading symbol makes sense, but the calculus is not as simple - in particular, there is usually no complete analogue of the relation $d d=0$.

To construct the zeta function and the heat operator of a differential operator $D$ on sections of $\mathbb{V}$, we make the following assumptions.

Analytic assumptions. $\left(M^{n}, g\right)$ is an $n$-dimensional compact smooth manifold, and $\mathbb{V}$ is a vector bundle over $M$ with a Hermitian metric $h . A$ is a smooth-coefficient differential operator of positive order on sections $\mathbb{V}$ which is formally self-adjoint:

$$
\int_{M} h(\varphi, A \psi) d v_{g}=\int_{M} h(A \varphi, \psi) d v_{g}
$$

and $A$ has positive definite leading symbol.

From these assumptions we may conclude that $A$ has even order $2 \ell$, since taking $\xi \mapsto-\xi$ reverses the sign of the leading symbol for odd-order operators. In addition, $L^{2}(M, \mathbb{V})$ has a complete orthonormal set $\left\{\varphi_{j}\right\}$ of eigensections for $A$ with real eigenvalues

$$
\lambda_{0} \leq \lambda_{1} \leq \cdots \leq \lambda_{j} \leq \cdots,
$$

satisfying

$$
\lambda_{j} \sim \text { const } \cdot j^{2 \ell / n}, \quad j \rightarrow \infty .
$$

(4) is the Weyl asymptotic law; see, e.g., [23]. 
If $r \geq 0$, the Sobolev space $L_{r}^{2}(M, \mathbb{V})$ is the domain of the operator

$$
\left(A-\lambda_{0}+1\right)^{r / 2 \ell}: \quad \sum_{j} c_{j} \varphi_{j} \mapsto \sum_{j} c_{j}\left(\lambda_{j}-\lambda_{0}+1\right)^{r / 2 \ell} \varphi_{j}
$$

in $L^{2}(M, \mathbb{V})$. The absence of any reference to $A$ in the notation $L_{r}^{2}(M, \mathbb{V})$ suggests that this domain is independent of $A$, and in fact it is. (As a consequence of the compactness of $M$, the Sobolev spaces do not even depend on the metric of the tangent bundle or of $\mathbb{V}$; see [28].) In addition, there is always some usable operator $A$ - for example, make metric and connection choices and take the Laplacian $\nabla^{*} \nabla$. Though the Sobolev space $L_{r}^{2}(M, \mathbb{V})$ is Hilbertizable, it is more productive to think of it as a Banach space, with the equivalence class of norms represented by

$$
\|\varphi\|^{2}=\int_{M} h\left(\left(A-\lambda_{0}+1\right)^{r / 2 \ell} \varphi,\left(A-\lambda_{0}+1\right)^{r / 2 \ell} \varphi\right) d v_{g}
$$

This is just a more-or-less random choice of a norm from the relevant equivalence class. When delicate invariance considerations are in play, it sometimes pays to pick an invariant representative of this class; see, for example, the last section below.

The dual space is denoted $L_{-r}^{2}(M, \mathbb{V})$; for real numbers $r \leq s$,

$$
\begin{aligned}
(\text { distributional sections })= & \mathcal{E}(M, \mathbb{V}) \subset L_{r}^{2}(M, \mathbb{V}) \subset L_{s}^{2}(M, \mathbb{V}) \\
& \subset C^{\infty}(M, \mathbb{V})=(\text { smooth sections }),
\end{aligned}
$$

and $L^{2}=L_{0}^{2}$. The Sobolev Lemma shows that

$$
C^{\infty}=\bigcap_{r} L_{r}^{2}
$$

and this, together with the estimates defining the space of distributions, shows that

$$
\mathcal{E}=\bigcup_{r} L_{r}^{2}
$$

The heat operator $e^{-t A}, t>0$, is of trace class, and is smoothing: it carries $L_{r}^{2}$ continuously to $L_{s}^{2}$ for any $r, s$, and thus carries distributional sections to to smooth sections. As a result, composition with $e^{-t A}$ has a very "civilizing" effect on other operators: if, for some $N$, the operator $B$ carries $L_{r}^{2}$ to $L_{r-N}^{2}$ for each $r$, then $B e^{-t A}$ and $e^{-t A} B$ carry distributional sections to smooth sections.

The heat trace has small-time asymptotics:

$$
\operatorname{Tr}_{L^{2}} e^{-t A} \sim a_{0}[A] t^{\frac{-n}{2 \ell}}+a_{1}[A] t^{\frac{-n+1}{2 \ell}}+\cdots+a_{k}[A] t^{\frac{-n+k}{2 \ell}}+\cdots, \quad t \downarrow 0 .
$$

This means that if we take the sum on the right out to the $k^{\underline{t h}}$ term, we have accuracy to the order of the next term: $O\left(t^{(-n+k+1) / 2 \ell}\right)$.

Note that one can conclude this accuracy immediately from a much weaker statement accuracy to order $O\left(t^{N(k)}\right)$, where $N(k) \rightarrow \infty$ as $k \rightarrow \infty$. To see this, pick $k_{1}$ so that $N\left(k_{1}\right) \geq$ $(-n+k+1) / 2 \ell$, and expand out to order $k_{1}$. Then the error in approximating $\operatorname{Tr}_{L^{2}} e^{-t A}$ by

$$
a_{0} t^{\frac{-n}{2 \ell}}+a_{1} t^{\frac{-n+1}{2 \ell}}+\cdots+a_{k} t^{\frac{-n+k}{2 \ell}}
$$

is

$$
a_{k+1} t^{(-n+k+1) / 2 \ell}+\cdots+a_{k_{1}} t^{\left(-n+k_{1}\right) / 2 \ell}+O\left(t^{(-n+k+1) / 2 \ell}\right)=O\left(t^{(-n+k+1) / 2 \ell}\right) .
$$


The way in which the $a_{k}$ depend on (the total symbol of) $A$ can be minutely analyzed, and it is useful to do so. One qualitative observation is that the $a_{\text {odd }}$ vanish in the current setting. (On manifolds with boundary, they consist entirely of boundary integrals.) Again, an excellent reference is [23].

In addition to the asymptotic series above, the heat operator trace also expands as a Dirichlet series of the first kind,

$$
\operatorname{Tr}_{L^{2}}\left(e^{-t A}\right)=\sum_{j=0}^{\infty} e^{-\lambda_{j} t} \quad(t>0) .
$$

For example, when $A$ is the operator $-d^{2} / d \theta^{2}$ on the circle, the series is

$$
1+\sum_{j=1}^{\infty} 2 e^{-j^{2} t}
$$

The corresponding Dirichlet series of the second kind take the form

$$
\sum_{j=0}^{\infty} \lambda_{j}^{-s}
$$

Actually, this is not entirely accurate, since (4) allows a finite number of $\lambda_{j}$ to be negative, and a finite number to be 0 . Some sort of artificial "fix" is thus needed to write such a Dirichlet series; we shall make the choice

$$
\sum_{\lambda_{j} \neq 0}\left|\lambda_{j}\right|^{-s}
$$

this is the zeta function $\zeta_{A}(s)$. Whatever artificial convention we choose for dealing with nonpositive eigenvalues, the effects will be felt in all succeeding formulas which make contact with the zeta function.

The two types of Dirichlet series are related by the Mellin transform

$$
(\mathcal{M} F)(s)=\frac{1}{\Gamma(s)} \int_{0}^{\infty} t^{s-1} F(t) d t
$$

under which

$$
\mathcal{M}: e^{-\lambda t} \mapsto \lambda^{-s} \quad(\lambda>0) .
$$

Because of (5),

$$
\mathcal{M}: \underbrace{\operatorname{Tr} e^{-t A}}_{=: Z_{A}(t)} \mapsto \underbrace{\operatorname{Tr} A^{-s}}_{\zeta_{A}(s)}
$$

when $A$ has positive spectrum. When there are nonpositive eigenvalues, the convention on $\zeta_{A}(s)$ adopted above forces us to replace $Z_{A}(t)$ by

$$
\tilde{Z}_{A}(t)=\sum_{\lambda_{j} \neq 0} e^{-t\left|\lambda_{j}\right|}=Z_{A}(t)-q[A]+2 \sum_{\lambda_{j}<0} \sinh \left(\lambda_{j} t\right)
$$

where $q[A]$ is the multiplicity of 0 as an eigenvalue of $A$.

The analytic continuation of the zeta function, which so far is only well-defined on $\operatorname{Re} s>n / 2 \ell$ (by the Weyl asymptotics (4)), is accomplished by substituting the small-time heat asymptotics 
into the Mellin transform expression for the zeta function. More precisely, assuming for the moment that there are no nonpositive eigenvalues,

$$
\Gamma(s) \zeta_{A}(s)=\sum_{k=0}^{N} a_{k}[A]\left(s-\frac{n-k}{2 \ell}\right)^{-1}+\underbrace{\int_{0}^{1} t^{s-1} O\left(t^{(-n+k+1) / 2 \ell}\right) d t}_{\text {regular, Re } s>\frac{n-k}{2 \ell}}+\underbrace{\int_{1}^{\infty} t^{s-1} \sum_{j} e^{-t \lambda_{j}} d t}_{\text {entire }} .
$$

Since we can do this for arbitrarily large $N$, the $\Gamma \zeta$ function has been analytically continued to a meromorphic function with possible simple poles at the $(n-k) / 2 \ell$, where $k$ is an even natural number. A useful viewpoint is that we are "approximating" the $\Gamma \zeta$ function; the error is "small" when it is regular on a half plane starting far to the left. In this sense the "smallest" functions are the entire ones.

To paraphrase the above, the $\Gamma \zeta$ function has at most simple poles on the real axis, at equal decrements starting with $s=n / 2 \ell$. Thus the zeta function has no poles at nonpositive integers they're resolved by the zeros of $1 / \Gamma(s)$. In particular,

$$
\zeta_{A}(s) \text { is regular at } s=0 .
$$

This is what we needed in order to state the zeta function definition of the determinant,

$$
\operatorname{det} A=e^{-\zeta_{A}^{\prime}(0)} \text {. }
$$

Remark 1. The regularity of the zeta function at $s=0$ is a more delicate property than the Weyl asymptotics (4). It is possible to construct sequences of $\lambda_{j}$ satisfying the Weyl asymptotics for which the poles of the corresponding zeta functions perform various tricks - in particular, one can place a pole at $s=0$. Somehow, regularity at $s=0$ indicates that an "organic" property of differential (or pseudo-differential) operators is being detected by the zeta function.

Remark 2. If we don't necessarily have positive eigenvalues, the new spectral function $\tilde{Z}_{A}(t)$ of (6) has its own modified heat coefficients $\tilde{a}_{k}$, defined by

$$
-q[A]+2 \sum_{\lambda_{j}<0} \sinh \left(t \lambda_{j}\right)+\sum_{k=0}^{\infty} a_{k}[A] t^{(k-n) / 2} \sim \sum_{k=0}^{\infty} \tilde{a}_{k}[A] t^{(k-n) / 2} .
$$

Note that $\tilde{a}_{k}=a_{k}$ for $k<n$. To build more determinant-like properties into the functional determinant, we might insert a sign to better monitor the negative eigenvalues:

$$
\operatorname{det} A:=(-1)^{\#\left\{\lambda_{j}<0\right\}} e^{-\zeta_{A}^{\prime}(0)} \text {. }
$$

\section{Variation of the functional determinant}

We now begin to imagine wiggling the operator $A$ in various ways. For example, we could let the operator $A$ depend on the geometry (i.e. the metric $g$ ) like the Laplacian does, and vary the metric. An important way to vary the metric is conformally:

$$
\bar{g}=\Omega^{2} g, \quad 0<\Omega \in C^{\infty}(M) .
$$

In fact, by writing

$$
g_{\omega}=e^{2 \omega} g_{0},
$$

we can parameterize the conformal class of $g_{0}$ by $\omega \in C^{\infty}(M)$. 
There are differential operators that are well adapted to this variation, namely the conformal covariants, or conformally invariant differential operators. Such an "operator" is really a rule assigning operators to metrics in a natural way, and has

$$
\bar{g}=\Omega^{2} g \Rightarrow \bar{D} \varphi=\Omega^{-b} D\left(\Omega^{a} \varphi\right)
$$

for some conformal biweight $(a, b)$ (and all sections $\varphi$ of the appropriate bundle). By rephrasing things in terms of operators on density bundles of appropriate weights, we could restate this as $\overline{D^{\prime}}=D^{\prime}$, for a uniquely determined operator $D^{\prime}$, but we shall not pursue that viewpoint here. The best-known example of a conformal covariant is the conformal Laplacian

$$
Y=\Delta+\frac{n-2}{4(n-1)} K \quad(K=\text { scalar curvature })
$$

on scalar functions; its conformal biweight is $((n-2) / 2,(n+2) / 2)$.

The infinitesimal form of the conformal covariance relation is equivalent to the finite form (7), and is sometimes more useful. Let the metric run through the curve $\left\{g_{\varepsilon \omega}\right\}_{\varepsilon \in \mathbb{R}}$ within a conformal class, and let

$$
\text { - }=\left.\frac{d}{d \varepsilon}\right|_{\varepsilon=0} .
$$

Then a conformal covariant has

$$
D^{\bullet} \varphi=a D(\omega \varphi)-b \omega D \varphi
$$

or, in operator terms,

$$
D^{\bullet}=-(b-a) \omega D+a[D, \omega] .
$$

(The final $\omega$ in this formula should be interpreted as multiplication by $\omega$.) Infinitesimal conformal covariance is equivalent to finite conformal covariance, since any metric $g_{\omega}$ in the conformal class may be connected to $g_{0}$ by the curve $\left\{g_{\varepsilon \omega}\right\}_{\varepsilon \in[0,1]}$.

In addition to the metric $g$, there are other standard ingredients involved in some geometric operators - the volume form $E$, and/or the fundamental tensor-spinor $\gamma$. If these are used, they should be scaled compatibly in forming $\bar{D}$ :

$$
\bar{E}=\Omega^{n} E, \quad \bar{\gamma}=\Omega^{-1} \gamma .
$$

An example of a conformal covariant for which this is relevant is the Dirac operator $\not \nabla=\gamma^{a} \nabla_{a}$ on spinors. its conformal biweight is $((n-1) / 2,(n+1) / 2)$. Another example is the operator $\star d$ on $(n-1) / 2$-forms in odd dimensions; here the Hodge star operator carries $p$-forms to $(n-p)$-forms on an oriented manifold, and is defined by

$$
(\star \varphi)_{a_{p+1} \ldots a_{n}}=\frac{1}{p !} E_{a_{1} \ldots a_{n}} \varphi^{a_{1} \ldots a_{p}} .
$$

This makes $E$ enter the definition of $\star d$ on $\Lambda^{(n-1) / 2}$; the conformal biweight of this operator is $(0,2)$.

We would like to know what happens to our spectral data if we vary conformally, and if our operator $A$ depends on $g$ in a conformally "nice" way. It is generally hopeless to try to track the motion of individual eigenvalues (except in very special circumstances; see the discussion after (10) below). However, it is sometimes possible to track the motion of quantities like the heat coefficients $a_{k}$ and the functional determinant. The reason is that these quantities have natural definitions in terms of traces of operators; such definitions take account of the whole 
spectrum. If the variations of the operators are known, there is some chance of computing the variation of the natural spectral quantity. As we shall see, conformal variation of suitable operators yields a setting in which variational computations can be done.

The good class of suitable operators is given by:

Conformal assumptions. $A=D^{h}$ is a positive power of a positive-order conformal covariant $D$.

For example, the conformal Laplacian satisfies both the analytic and conformal assumptions, since it is itself a conformal covariant. The Dirac operator $\nabla$ is conformally covariant, but does not have positive definite leading symbol. However its power $\nabla^{2}$, though not conformally covariant, has positive definite leading symbol (in fact, leading symbol $|\xi|^{2} \mathrm{Id}$ ), and so satisfies our analytic and conformal assumptions. The operator $\star d$ on $\Lambda^{(n-1) / 2}$ does not have invertible leading symbol, so there is no hope of any of its powers satisfying the analytic assumptions.

Under our analytic and conformal assumptions, we have the following:

Theorem 1 (Theorem on Variations [15, 16, 30, 20]). With notation as explained just below,

$$
\begin{aligned}
& a_{k}[A]^{\bullet}=(n-k) a_{k}[\omega, A]=(n-k) \int_{M} \omega U_{k}[A] d v_{g_{0}}, \\
& \zeta_{A}^{\prime}(0)^{\bullet}=2 \ell \int_{M} \omega\left\{U_{n}[A]-\sum_{\lambda_{j}=0}\left|\varphi_{j}\right|^{2}\right\} d v_{g_{0}},
\end{aligned}
$$

where $\left\{\varphi_{j}\right\}$ is any orthonormal basis of the 0-eigenspace of $A$.

The [30] and [20] references really just treat the conformal Laplacian.

The statement uses the local heat coefficients $a_{k}[\omega, A]$ and $U_{k}[A]$. The first of these, $a_{k}[\omega, A]$ is a term in the small-time asymptotics of $\operatorname{Tr}_{L^{2}} \omega e^{-t A}$ :

$$
\operatorname{Tr}_{L^{2}} \omega e^{-t A} \sim a_{0}[\omega, A] t^{\frac{-n}{2 \ell}}+a_{1}[\omega, A] t^{\frac{-n+1}{2 \ell}}+\cdots+a_{k}[\omega, A] t^{\frac{-n+k}{2 \ell}}+\cdots, \quad t \downarrow 0 .
$$

An analysis of the dependence of the heat coefficients $a_{k}[\omega, A]$ on $A$ and $\omega$ shows that they are integrals of differential polynomials in the total symbol of $A$, with coefficients that depend (in a universal way) smoothly on the leading symbol and linearly on the jets of $\omega$. As a result, after integration by parts,

$$
a_{k}[\omega, A]=\int_{M} \omega U_{k}[A] d v_{g}
$$

for some functions $U_{k}[A]$ built in a universal way from the total symbol of $A$.

In particular, we have:

Theorem 2 (Conformal Index Theorem [15]). Under the above assumptions, the coefficient $a_{n}[A]$ is conformally invariant.

Some other conformally invariant quantities which are important in these calculations are

$$
q[A]=\operatorname{dim} \mathcal{N}(A)=\#\left\{\lambda_{j}=0\right\}, \quad \zeta_{A}(0)=a_{n}[A]-q[A], \quad \#\left\{\lambda_{j}<0\right\} .
$$

The invariance of $q[A]$ is immediate from the conformal covariance relation. The invariance of $\#\left\{\lambda_{j}<0\right\}$ then follows from this and Browder's Theorem [18]. At the metric $g_{0}$, for some $\delta>0$, the negative eigenvalues are contained in the interval $(-\infty,-\delta)$, and the positive ones in $(\delta, \infty)$. With the invariance of the number of zero eigenvalues, this is good enough to keep the number 
of negative eigenvalues constant on $\left\{g_{\varepsilon \omega}\right\}$, for fixed $\omega$ and $|\varepsilon|$ bounded by some $\varepsilon_{0}>0$. But this in turn implies constancy on the whole conformal class.

The local data $a_{k}[\omega, A], U_{k}[A]$ carry more information than $a_{k}[A]$; in fact

$$
a_{k}[A]=a_{k}[1, A]=\int U_{k} .
$$

This more refined data (involving $\omega$ ) is also spectral, but the spectra of many more operators (than just $A$ ) are now involved.

If $A$ is natural, that is, if it is built in a universal way from the metric (and possibly $E$ and/or $\gamma$ ), then the total symbol of $A$ must also be. But then, in turn, the $U_{k}[A]$ are also built in this way. By Weyl's invariant theory [37], the $U_{k}[A]$ are built polynomially from the Riemann curvature and its iterated covariant derivatives.

For example, if $K$ is the scalar curvature and $\Delta$ is the Laplacian on functions,

$$
\begin{aligned}
& U_{0}[\Delta+a K]=(4 \pi)^{-n / 2}, \\
& U_{2}[\Delta+a K]=(4 \pi)^{-n / 2}\left(\frac{1}{6}-a\right) K, \\
& U_{4}[\Delta+a K]=(4 \pi)^{-n / 2} \cdot \frac{1}{180} \cdot\left[90\left(\frac{1}{6}-a\right)^{2} K^{2}-|r|^{2}+|R|^{2}-30\left(\frac{1}{5}-a\right) \Delta K\right],
\end{aligned}
$$

where $R$ (resp. $r$ ) is the Riemann (resp. Ricci) tensor. One reason that formulas like this are important is hinted at by (9): we might be able to parlay explicit knowledge of $U_{n}[A]$ into information about det $A$ as a function on the conformal class $\left\{g_{\omega}=e^{2 \omega} g_{0}\right\}$. In fact, there are two genuinely different ways of doing this, one based on (9), and one based on both (9) and (8).

The first method is to simply integrate the variation along the curve $\left\{g_{\varepsilon \omega}\right\}$, starting from $g_{0}$ and ending at $g_{\omega}$. As it turns out, the homogeneities are such that this is just the integration of a polynomial in $\varepsilon$. Specifically,

$$
U_{k}\left[A_{\omega}\right]=e^{-2 k \omega}\left(U_{k}\left[A_{0}\right]+U_{k}^{(1)}\left[A_{0}\right](\omega)+\cdots+U_{k}^{(k)}\left[A_{0}\right](\omega)\right),
$$

where $U_{k}^{(i)}$ is a polynomial in the Riemann curvature and $\omega$ and their covariant derivatives, and is of homogeneous degree $i$ in $\omega$. (In fact, it depends only on $d \omega$; see [6].) Here we have adopted the convention of labelling geometric objects depending on the metric by the subscript $\omega$, rather than the subscript $g_{\omega}$; for example,

$$
A_{\omega}=A_{g_{\omega}} .
$$

On the other hand,

$$
d v_{\omega}=\varepsilon^{n \omega} d v_{0} .
$$

As a result, $U_{n}\left[A_{\varepsilon \omega}\right] d v_{\varepsilon \omega}$ is polynomial in $\varepsilon$, of degree at most $n$.

(By the conformal invariance of the conformal index $\int U_{n}[A] d v$, we may actually say "of degree at most $n-1$ ". If $n$ is odd, $U_{n}[A]$ is identically zero, while if $n$ is even, homogeneity considerations force the $U_{n}^{(n)}[A]$ term in (12) to take the form $c \cdot|d \omega|^{n}$, for some universal constant $c$. But if $c$ were nonzero, a simple scaling argument in $\omega$ shows that the conformal index is unbounded on the conformal class, contradicting the fact that it is constant.)

The second method is an adaptation of the physicists' dimensional regularization. For simplicity in this discussion, suppose that $A$ has no zero modes (eigenvalues) at $g_{0}$ (and thus on the whole conformal class). Suppose that we are interested in the functional determinant in dimension $n$. The idea is to get a formula for $a_{n}[A]$ which is valid in an infinite number of dimensions $n^{\prime}$, including $n^{\prime}=n$. Denote by Prim (for "primitive") the conformal anti-variation 
corresponding to the conformal variation - above; this is well-defined up to a "constant of integration" - a constant function on the conformal class. The Conformal Index Theorem then says that

$$
\operatorname{Prim} \int \omega U_{n}[A]= \begin{cases}\frac{a_{n}[A]}{n^{\prime}-n}+\text { const, } \\ \frac{1}{2 \ell} \zeta_{A}^{\prime}(0)+\text { const, } & n^{\prime} \neq n, \\ n^{\prime}=n .\end{cases}
$$

The idea is now that the first formula continues to the second. To standardize the constants of integration, we view the primitives as functions of an indeterminate conformal factor $\omega$, measured from a base metric $g_{0}$, and require that our primitives vanish at $g_{0}$ :

$$
\operatorname{Prim}_{0} \int \omega U_{n}[A]= \begin{cases}\frac{a_{n}\left[A_{\omega}\right]-a_{n}\left[A_{0}\right]}{n^{\prime}-n}, & n^{\prime} \neq n, \\ \frac{1}{2 \ell}\left(\zeta_{A_{\omega}}^{\prime}(0)-\zeta_{A_{0}}(0)\right), & n^{\prime}=n .\end{cases}
$$

The analytic continuation to the special dimension $n^{\prime}=n$ is then expressed by the formula

$$
\left.\frac{a_{n}\left[A_{\omega}\right]-a_{n}\left[A_{0}\right]}{n^{\prime}-n}\right|_{n^{\prime}=n}=\frac{1}{2 \ell}\left(\zeta_{A_{\omega}}^{\prime}(0)-\zeta_{A_{0}}^{\prime}(0)\right) .
$$

As a result, we only need to know the integrated invariant $a_{n}[A]$, not the whole local invariant $U_{n}[A]$. But we need to know it in "all" dimensions, or at least an infinite number of dimensions. If we have this information, we get a formula for the difference of log-determinants at two conformal metrics; that is, the quotient of determinants.

Note that the first method (fixing the dimension and integrating along a curve in the conformal class) is also giving us determinant quotients, since the definite integral described above computes $\zeta_{A_{\omega}}^{\prime}(0)-\zeta_{A_{0}}^{\prime}(0)$.

Of course, one cannot continue a formula from an infinite number of values of the independent variable to a special value. But such a continuation is possible in the case of a rational formula. That is, a rational function on $\mathbb{C}$ may be continued from any infinite set of values, because a polynomial function can. Alternatively, a meromorphic function on $\mathbb{C}$ for which $\infty$ is not an essential singularity may be continued from any set having $\infty$ (or any other point of the Riemann sphere, for that matter) as a limit point. What we need is a way of phrasing the above statements in terms of linear combinations, with rational coefficients, of a stable (under change of dimension) basis of local invariants. The ingredients out of which these invariants will be built will sometimes include the indeterminate conformal factor $\omega$-like the metric or the Riemann curvature, it will be thought of as just another formal variable, rather than a specific function on a specific manifold.

Of course one knows that the heat invariants are usually not rational in the dimension. For example, for the operators $\Delta+a K$ on scalar functions of (11), it looks as if $U_{k}[A]$ will have the form $(4 \pi)^{-n / 2} V_{k}[A]$ with $V_{k}[A]$ rational in $n$ (as long as the constant $a=a(n)$ is rational in $n$ ). This is in fact true for all $k$, not just the $k \leq 4$ cases in (11). But the exponential function $(4 \pi)^{-n / 2}$ certainly has an essential singularity at $n=\infty$. The remedy for this is the fact that the $(4 \pi)^{-n / 2}$ factor is universal - one can factor it out of the coefficient of each invariant, and it's constant with respect to the relevant variation of the geometry. Thus it's really the coefficients of $V_{k}[A]$ (and related quantities) that we're talking about, and these coefficients are rational. The possibility of factoring out a universal function of $n$ so that we are left with rational functions of $n$ is really a question about the form of the dependence of the operator's leading symbol on the metric. Similar results hold for other leading symbols; for example $|\xi|^{4}[10]$, and bundle-valued symbols which are not just powers of $|\xi|^{2}$ times the identity [3]. 
Demonstration of the Theorem on Variations. The key to the variational formulas is the conformal variation of the heat operator trace, or of the zeta function:

$$
\begin{aligned}
Z(t)^{\bullet} & =\left(\operatorname{Tr} e^{-t A}\right)^{\bullet}=-t \operatorname{Tr} A^{\bullet} e^{-t A}=-t \operatorname{Tr} \underbrace{D^{\bullet}}_{a D \omega-b \omega D} h D^{h-1} \exp \left(-t D^{h}\right) \\
& =t h(b-a) \operatorname{Tr} \omega D^{h} \exp \left(-t D^{h}\right) .
\end{aligned}
$$

Note that the step $\left(\operatorname{Tr} e^{-t A}\right)^{\bullet}=-t \operatorname{Tr} A^{\bullet} e^{-t A}$ is not valid on the operator level, even formally; the operators involved only have the same trace. There is no problem with domains due to the "civilizing" effect of the smoothing operator $\exp (-t A)$ - placing it next to a pseudo-differential operator of finite order produces an operator of order $-\infty$, and a fortiori one that is bounded on $L^{2}$. By homogeneity considerations,

$$
h(b-a)=2 \ell,
$$

so

$$
\left(\operatorname{Tr} e^{-t A}\right)^{\bullet}=2 \ell t \operatorname{Tr} \omega A e^{-t A}=-2 \ell t \frac{d}{d t} \operatorname{Tr} \omega e^{-t A} .
$$

However,

$$
t \frac{d}{d t}\left(t^{(k-n) / 2 \ell}\right)=\frac{k-n}{2 \ell} t^{(k-n) / 2 \ell} .
$$

So, provided that term-by-term variation of the asymptotic series is justified (see the remarks below),

$$
a_{k}^{\bullet}[A]=(n-k) a_{k}[\omega, A] .
$$

From the zeta function point of view, the variational calculation is

$$
\begin{aligned}
\Gamma(s) \zeta(s)^{\bullet} & =\Gamma(s)\left(\operatorname{Tr} A^{-s}\right)^{\bullet}=\Gamma(s)\left(\operatorname{Tr} D^{-h s}\right)^{\bullet}=\Gamma(s) \operatorname{Tr} D^{\bullet}(-h s) D^{-h s-1} \\
& =(b-a) h s \Gamma(s) \operatorname{Tr} \omega D^{-h s}=2 \ell s \Gamma(s) \zeta(\omega, s) .
\end{aligned}
$$

But $a_{k}$ is the residue of the $\Gamma \zeta$ function at $(n-k) / 2 \ell$, so we recover (13).

In addition, assuming for the moment that 0 is not an eigenvalue of $A$,

$$
\zeta_{A}^{\prime}(0)^{\bullet}=\left.2 \ell \frac{d}{d s}\right|_{s=0}\left(s \zeta_{A}(\omega, s)\right)=2 \ell \zeta_{A}(\omega, 0) .
$$

But $\zeta_{A}(\omega, 0)=a_{n}[\omega, A]$, since $\operatorname{Res}_{s=0} \Gamma(s)=1$. If 0 is an eigenvalue for $A$, we carry out the last calculations after replacing $\exp (-t A)$ and $A^{-s}$ by the restrictions of $\exp (-t|A|)$ and $|A|^{-s}$ to the orthogonal complement in $L^{2}$ of $\mathcal{N}(A)$, the null space of $A$. (Here a "restriction" is extended back to the whole space as 0 on $\mathcal{N}(A)$.) On the level of kernel functions, we are then dealing with

$$
\sum_{\lambda_{j} \neq 0} \exp \left(-\left|\lambda_{j}\right| t\right) \varphi_{j} \otimes \varphi_{j}^{*} \quad \text { and } \quad \sum_{\lambda_{j} \neq 0}\left|\lambda_{j}\right|^{-s} \varphi_{j} \otimes \varphi_{j}^{*}
$$

The zeta function arises from such a modified kernel, while the $Z$-function (as reflected in the $\left.U_{k}[A]\right)$ arises from the "original" kernel function. Chasing through the consequences of this mismatch gives rise to the extra term in (9).

In both the $Z$-function and zeta function arguments for the variational formula (8), there is an interchange of limit operations. In the case of the $Z$ function, we are claiming that a certain 
asymptotic series may be differentiated term-by-term with respect to an outside parameter (the $\varepsilon$ in the conformal factor $e^{\varepsilon \omega}$ ). That is, we are asserting that the asymptotics of the variation are the variation of the asymptotics. In the zeta function argument, an equivalent strong assertion is made: that the analytic continuation of the variation (again, with respect to the outside parameter $\varepsilon$ ) is the variation of the analytic continuation. This is not just a matter of the uniqueness of the analytic continuation. In general, families of holomorphic functions with extremely nice dependence on an outside parameter may exhibit very ugly dependence in their analytic continuations. In either case, the issue comes down to suitable uniformity in the outside parameter of the estimates of error in the asymptotic expansion - in the zeta function argument, this enters when the heat operator and the Mellin transform are used to accomplish the analytic continuation.

It's also worth noting that the issue of term-by-term differentiation of the asymptotic series with respect to an outside parameter (here, $\varepsilon$ ) is more delicate than term-by-term differentiation with respect to the asymptotic parameter $t$. Indeed, since one can trivially integrate term by term, one can also differentiate: if

$$
f(t) \sim \sum_{k} a_{k} t^{k}, \quad f^{\prime}(t) \sim \sum_{k} b_{k} t^{k} \quad \text { with } \quad b_{0}=0,
$$

then

$$
f(t)+\text { const } \sim \sum_{k} \frac{b_{k}}{k+1} t^{k+1},
$$

so that

$$
a_{k+1}=\frac{b_{k}}{k+1}, \quad k \neq-1 .
$$

This shows that the series for $f^{\prime}(t)$ is the term-by-term derivative of that for $f(t)$, since the derivative of the term $a_{0} t^{0}$ is 0 .

Note that the determinant quotient problem for conformal metrics is more or less trivial in odd dimensions (at least for manifolds without boundary), since $U_{n}[A]=0$. (As noted earlier, the $a_{\text {odd }}$ vanish; the stronger statement that the $U_{\text {odd }}$ vanish is also true.) This means that the only variation in the determinant comes from the $\varphi_{j}$ terms in (9).

Here's a sample calculation to illustrate the dimensional regularization method of computing the determinant quotient. Our "input" operator $A$ will be the square of the Dirac operator $\nabla=$ $\gamma^{a} \nabla_{a}$ on the bundle of spinors. (Recall the discussion just before the Theorem on Variations.) Suppose we would like the determinant quotient in dimension 2. Since

$$
a_{2}\left[\nabla^{2}\right]=-\frac{1}{12} 2^{[n / 2]}(4 \pi)^{-n / 2} \int K,
$$

the determinant quotient will be

$$
\left[\frac{1}{n-2} \int\left((K d v)_{\omega}-(K d v)_{0}\right)\right]_{n=2} .
$$

If this is to work, there must somehow be a "hidden" $n-2$ factor in the integral. To bring this out, recall the Yamabe equation

$$
Y u:=\Delta u+\frac{n-2}{4(n-1)} K u=\frac{n-2}{4(n-1)} \bar{K} u^{\frac{n+2}{n-2}}
$$

for the scalar curvature after a conformal change

$$
\bar{g}=\Omega^{2} g, \quad 0<\Omega \in C^{\infty}, \quad u=\Omega^{\frac{n-2}{2}} .
$$


(Equation (15) is actually the conformal covariance relation for the conformal Laplacian, applied to the function 1.) Rewrite (15) as

$$
\begin{aligned}
& \Delta\left(e^{\frac{n-2}{2} \omega}\right)+\frac{n-2}{4(n-1)} K e^{\frac{n-2}{2} \omega}=\frac{n-2}{4(n-1)} \bar{K} e^{\frac{n+2}{2} \omega}, \\
& \Delta_{0}\left(e^{\frac{n-2}{2} \omega}-1\right)+\frac{n-2}{4(n-1)} K_{0} e^{\frac{n-2}{2} \omega}=\frac{n-2}{4(n-1)} K_{\omega} e^{\frac{n+2}{2} \omega} .
\end{aligned}
$$

On the second line, we have introduced a gratuitous -1 into the argument of $\Delta_{0}$; this is harmless because $\Delta_{0} 1=0$. It is useful because it allows us to divide by $(n-2) / 2$ :

$$
\Delta_{0} f\left(\frac{n-2}{2} \omega\right)+\frac{1}{2(n-1)} K_{0} e^{\frac{n-2}{2} \omega}=\frac{1}{2(n-1)} K_{\omega} e^{\frac{n+2}{2} \omega}
$$

where

$$
f(x):=\frac{e^{x}-1}{x} .
$$

Evaluation at $n=2$ now yields the Gauss curvature prescription equation

$$
\Delta_{0} \omega+\frac{1}{2} K_{0}=\frac{1}{2} K_{\omega} e^{2 \omega} \quad(n=2) .
$$

In particular,

$$
\int(K d v)_{0}=\int(K d v)_{\omega} \quad(n=2)
$$

since the difference of the two integrands, $2\left(\Delta_{0} \omega\right) d v_{0}$, integrates to zero by the Divergence Theorem.

(17) just expresses the invariance of the conformal index in this setting. To get a handle on (14) we need to go to higher order in the above calculation, in the dimension parameter

$$
\beta=\frac{n-2}{2} \text {. }
$$

For this, first multiply (16) by $d v_{0}=e^{-n \omega} d v_{\omega}$ :

$$
\left\{\Delta_{0}\left(\frac{e^{\beta \omega}-1}{\beta}\right)+\frac{1}{2(n-1)} K_{0} e^{\beta \omega}\right\} d v_{0}=\frac{1}{2(n-1)} K_{\omega} e^{-\beta \omega} d v_{\omega},
$$

Now multiply by $\exp (\beta \omega)$ :

$$
\left\{e^{\beta \omega} \Delta_{0}\left(\frac{e^{\beta \omega}-1}{\beta}\right)+\frac{1}{2(n-1)} K_{0} e^{2 \beta \omega}\right\} d v_{0}=\frac{1}{2(n-1)}(K d v)_{\omega} .
$$

If we now set

$$
J:=\frac{K}{2(n-1)},
$$

the above can be rewritten as

$$
\begin{aligned}
(J d v)_{\omega}-(J d v)_{0} & =e^{\beta \omega} \Delta_{0}\left(\frac{e^{\beta \omega}-1}{\beta}\right)+\left(e^{2 \beta \omega}-1\right)(J d v)_{0} \\
& =\Delta_{0}\left(\frac{e^{\beta \omega}-1}{\beta}\right)+\left(e^{\beta \omega}-1\right) \Delta_{0}\left(\frac{e^{\beta \omega}-1}{\beta}\right)+\left(e^{2 \beta \omega}-1\right)(J d v)_{0} .
\end{aligned}
$$


Now integrate and divide by $n-2$ :

$$
\frac{1}{2 \beta} \int\left((J d v)_{\omega}-(J d v)_{0}\right)=\int\left\{\frac{e^{\beta \omega}-1}{2 \beta} \Delta_{0}\left(\frac{e^{\beta \omega}-1}{\beta}\right)+\frac{e^{2 \beta \omega}-1}{2 \beta} J_{0}\right\} d v_{0} .
$$

Evaluating at $n=2$ (i.e., at $\beta=0$ ), this gives

$$
\left[\frac{1}{n-2} \int\left((J d v)_{\omega}-(J d v)_{0}\right)\right]_{n=2}=\int\left\{\frac{1}{2} \omega \Delta_{0} \omega+\omega J_{0}\right\} d v_{0} .
$$

As a result, if the Dirac operator does not take the eigenvalue 0 in the conformal class in which we are working,

$$
\zeta_{\nabla^{2}}^{\prime}(0)-\zeta_{\nabla_{0}^{2}}^{\prime 2}(0)=-\frac{1}{12 \pi} \int\left\{\frac{1}{2} \omega \Delta_{0} \omega+\omega J_{0}\right\} d v_{0}
$$

Note that the objects that appear in the final result, $\Delta$ and $J$, really appear just because of the form of the integrated invariant $a_{2}$. In fact, since the only local scalar invariant that can contribute to $a_{2}$ is $K$ (or $J$ ), the above formula is independent of $A$, except for the overall constant factor. For example, for the Laplacian on functions in dimension 2 ,

$$
\zeta_{\Delta_{\omega}}^{\prime}(0)-\zeta_{\Delta_{0}}^{\prime}(0)=\frac{1}{12 \pi} \int\left\{\frac{1}{2} \omega \Delta_{0} \omega+\omega J_{0}\right\} d v_{0}
$$

We compute this by extending $\Delta$ for $n=2$ to the conformal Laplacian $\Delta+(n-2) J / 2$ in higher dimensions.

\section{Extremals of the functional determinant}

When we begin to think about maximizing or minimizing the determinant, the first thing we notice is that it is not invariant under uniform scaling of the metric:

$$
\tilde{g}=e^{2 \alpha} g, \quad \alpha \in \mathbb{R} \quad \Rightarrow \quad \tilde{A}=e^{-2 \ell \alpha} A,
$$

so

$$
\zeta_{\tilde{A}}(s)=e^{2 \ell \alpha s} \zeta_{A}(s), \quad \zeta_{\tilde{A}}^{\prime}(0)=\zeta_{A}^{\prime}(0)+2 \ell \alpha \zeta_{A}(0) .
$$

Thus it's necessary to somehow penalize the determinant for the size of the metric. One way to do this is with a volume penalty:

$$
\mathcal{D}(A, g):=(\operatorname{vol} g)^{2 \ell \zeta_{A}(0) / n} \operatorname{det} A_{g}
$$

is invariant under uniform dilation. (Recall that $\zeta_{A}(0)$ is a conformal invariant.) The formula for the conformal variation of the determinant says that the conformal variation of the $\mathcal{D}$-functional is

$$
-\log \left(\mathcal{D}_{A}\right)^{\bullet}=2 \ell \operatorname{vol}(g)\left(\left(\omega U_{n}\right)^{\sim}-\tilde{\omega} \tilde{U}_{n}\right),
$$

where the tilde denotes the average value. Thus the $\mathcal{D}$-quotient is

$$
\log \frac{\mathcal{D}\left(A_{\omega}\right)}{\mathcal{D}\left(A_{0}\right)}=\frac{2 \ell \zeta_{A}(0)}{n} \log \underbrace{\frac{\int e^{n \omega} d v_{0}}{\int d v_{0}}}_{\left(e^{n \omega}\right)^{\sim}}+\log \frac{\operatorname{det}\left(A_{\omega}\right)}{\operatorname{det}\left(A_{0}\right)} .
$$


Now (to be somewhat vague) the coefficients in $\zeta_{A}(0)$ have something to do with the coefficients in the determinant term $-\zeta_{A}(0)$ is $a_{n}[A]$, and the determinant term somehow came from $a_{n}[A]$. As a result, though different $A$ produce different $\mathcal{D}$-functionals, combinations of a limited list of functionals keep appearing. For example in dimension 2, in the standard conformal class on the sphere $\mathbf{S}^{2}$, one is always looking at

$$
-\log \int_{\mathbb{S}^{2}} e^{2(\omega-\bar{\omega})} d \xi+\int_{\mathbb{S}^{2}} \omega\left(\Delta_{0} \omega\right) d \xi
$$

where $d \xi$ is the standard measure, normalized so that $\int_{\mathbf{S}^{2}} d \xi=1$. This is exactly the quantity that appears in the celebrated Moser-Trudinger inequality.

Recall the Sobolev embeddings

$$
L_{r}^{2} \hookrightarrow L^{2 n /(n-2 r)} .
$$

The inequalities asserting the boundedness of the inclusion operators have sharp forms (more on this later; see (28) below) on $\mathbf{S}^{2}$. What happens as $r \rightarrow n / 2$ ? One answer is the Sobolev lemma

$$
L_{\frac{n}{2}+\varepsilon}^{2} \hookrightarrow C^{0} .
$$

A deeper answer is the embedding

$$
L_{n / 2}^{2} \hookrightarrow e^{L} .
$$

The inequality describing this inclusion compares an $L_{n / 2}^{2}$ norm with a functional that governs admission to the Orlicz class $e^{L}$. When $n=2$, this is the Moser-Trudinger inequality; for other $n$, it is the exponential class inequality of Beckner [5] and Carlen-Loss [19]. The sharp form of the Moser-Trudinger inequality is:

\section{Theorem 3 (Moser-Trudinger inequality).}

$$
\log \int_{\mathbb{S}^{2}} e^{2(\omega-\bar{\omega})} d \xi \leq \int_{\mathbb{S}^{2}} \omega\left(\Delta_{0} \omega\right) d \xi
$$

with equality iff $e^{\omega}$ is a constant multiple of a conformal factor $\Omega_{h}$ on $\mathbb{S}^{2}$.

To explain the term conformal factor as it's being used here, recall that there is a Möbius group of conformal transformations of the Riemann sphere. In terms of Riemannian geometry, such a transformation (diffeomorphism) has

$$
\left(h^{-1}\right)^{*} g_{0}=\Omega_{h}^{2} g_{0} .
$$

The inequality is sharp because equality is attained for some $\omega$, and the case of equality is completely analyzed.

In 1982, Onofri [27] used the Moser-Trudinger inequality to prove that on $\mathbf{S}^{2}$, the volumepenalized determinant of $\Delta$ is maximized exactly at the standard metric and its diffeomorphic images. Part of this is special to 2 dimensions - each metric on $\mathbf{S}^{2}$ is diffeomorphic to one in the standard conformal class. Since diffeomorphisms preserve spectral invariants, it's therefore enough to study the standard conformal class. This is not the case in higher dimensions, even on the sphere.

In fact, the space which may be loosely described by

$$
\frac{\text { metrics }}{\operatorname{Diffeo}(M) \ltimes \operatorname{Conf}(M)}
$$


is just a point when $M=\mathbf{S}^{2}$. (The semidirect product $\ltimes$ in the denominator reflects the fact that diffeomorphisms act on conformal factors.) This space is generally a finite-dimensional object when $n=2$ (in fact, Teichmüller space), but it's a big, wild object when $n>2$.

One of the many questions that one can ask at this point is how all this plays out in higher dimensions - on $\mathbf{S}^{4}, \mathbf{S}^{6}$, and so on. Can we still solve a max/min problem by using sharp inequalties? What will the $\mathcal{D}$-quotient functional and the sharp inequalities look like? In the following paragraphs, we look at the objects and methods that will generalize the twodimensional versions appearing in the paragraph headings.

The Laplacian. The Laplacian $\Delta$ on scalar functions turned up in the formula for the determinant quotient in dimension 2, even if it was some "exotic" $A$ (like Dirac-squared) whose determinant we were measuring. In 1992, Graham, Jenne, Mason, and Sparling [25] proved the existence of a series of conformally covariant operators $P_{m}$, of order $m$ and of the form $\Delta^{m / 2}+$ (lower order); these operators exist for

all even $m$ when $n$ odd,

even $m \leq n$ when $n$ even.

The Paneitz operator $P_{4}$ was previously found in $[29,32,21]$.

In [39], Wünsch wrote a kind of formula for $P_{6}$ - really more of an algorithm for computing it, but enough to prove its existence.

The role of the 2-dimensional Laplacian in the determinant formulas is played by $P_{n}$ (the operator of dimension order), for even $n$. The role of the Gauss curvature is played by an invariant $Q_{n}$ (discovered in [16] in dimension 4, and in [8] in higher even dimensions) for which

$$
P_{m}=P_{m}^{0}+\frac{n-m}{2} Q_{m}, \quad P_{m}^{0} 1=0 .
$$

Dimensional regularization. The algebra of local invariants for general metrics can be simplified somewhat in the category of conformally flat metrics. These are characterized by the existence of local coordinates near each point in which the metric is $g_{a b}=\Omega^{2} \delta_{a b}$ for some smooth positive function $\Omega$. There is an alternative characterization, in terms of invariants: if $n \geq 4$, a metric is conformally flat if and only if its Weyl tensor vanishes. The Weyl tensor, in turn, may be characterized as the totally trace-free part of the Riemann curvature tensor. One has the following formulas. Let

$$
J=\frac{K}{2(n-1)}
$$

as above, and let

$$
V=\frac{r-J g}{n-2}
$$

where $r$ is the Ricci tensor. Then the Weyl tensor $C$ is

$$
C_{a b c d}=R_{a b c d}+V_{b c} g_{a d}-V_{b d} g_{a c}+V_{a d} g_{b c}-V_{a c} g_{b d} .
$$

As a consequence of the Bianchi identity, we have

$$
\nabla^{a} C_{a b c d}=(n-3)\left(\nabla_{c} V_{b d}-\nabla_{d} V_{b c}\right) .
$$

When $n=3$, the vanishing of $\nabla_{c} V_{b d}-\nabla_{d} V_{b c}$ characterizes conformal flatness (a fact which in itself is suggestive of some kind of dimensional regularization). In any case, for $n \geq 3$, in the conformally flat case, all local invariants are built from $g, V$ and $\nabla$ ( $J$ being an abbreviation 
for $V_{a}^{a}$ ), and one has the "extra identity" that $\nabla V$ is totally symmetric. The ideal of identities (i.e., the ideal of left sides of identities with right side 0) is generated by (20), together with the Ricci identities, which give formulas for $\left[\nabla_{a}, \nabla_{b}\right]$ applied to tensors of all ranks.

We shall concentrate on dimensional regularization in the conformally flat category, though the restriction is not essential - there is a perfectly good algebraic setting in the category of arbitrary metrics. However, conformally flat metrics provide the cleanest route to the very clean extremal results on the spheres $\mathbf{S}^{\text {even }}$.

Recall now our earlier formula for the determinant quotient:

$$
\frac{1}{2 \ell}\left(\zeta_{A_{\omega}}^{\prime}(0)-\zeta_{A_{0}}^{\prime}(0)\right)=\left.\frac{a_{n}\left[A_{\omega}\right]-a_{n}\left[A_{0}\right]}{n^{\prime}-n}\right|_{n^{\prime}=n},
$$

where $n$ is the fixed dimension (in which we want a formula), and $n^{\prime}$ is the "running" dimension. What one gets out of this for even $n>2$, given suitable dependence on $n^{\prime}$, is a formula for the penalized determinant quotient functional of the form

$$
C\left(\frac{n}{2\left(Q_{n}\right)_{0}} \int_{\mathbb{S}^{n}}\left(P_{n} \omega, \omega\right) d \xi-\log \int_{\mathbb{S}^{n}} e^{n(\omega-\bar{\omega})} d \xi\right)+\int_{\mathbb{S}^{n}}\left((B d \xi)_{\omega}-(B d \xi)_{0}\right),
$$

where $B=B[A]$ is some local scalar invariant, and $C$ is a constant.

The invariant $B$ is a linear combination of invariants from a precise (and remarkably short, for small $n$ ) list. For $n=2$, this list is empty; for $n=4$, there is one invariant that can contribute; for $n=6$, there are 3 , and for $n=8$, there are 8 .

Remark 3. Besides being a convenient tool for calculation, dimensional regularization produces a theoretical result on the nature of the functional, which may be paraphrased as follows. If one follows the other, fixed-dimension method of integrating along a curve in the conformal class, then after subtracting out the correct multiple of the functional

$$
\mathcal{F}_{0}(\omega):=\frac{n}{2\left(Q_{n}\right)_{0}} \int_{\mathbb{S}^{n}}\left(P_{n} \omega, \omega\right) d \xi-\log \int_{\mathbb{S}^{n}} e^{n(\omega-\bar{\omega})} d \xi,
$$

the dependence on $\omega$ of the remainder is that of a local invariant:

$$
\mathcal{F}(\omega):=\int_{\mathbb{S}^{n}}\left((B d \xi)_{\omega}-(B d \xi)_{0}\right) .
$$

We saw that in dimension 2, the penalized determinant quotient functional was an exactly the one estimated by the Moser-Trudinger inequality. What is the analogue for dimension 4, and higher dimensions? Remarkably, in the early 1990's, several lines of thought necessary for an understanding of this were maturing - higher dimensional Polyakov formulas [16], sharp inequalities [5, 19], and, though only an understanding of the Paneitz operator is needed in 4 dimensions, the GJMS operators [25].

The sharp exponential class inequality in $n$ dimensions is:

Theorem 4 (Exponential class inequality). The quantity

$$
\frac{n}{2 \Gamma(n)} \int_{\mathbb{S}^{n}}\left(\left(P_{n}\right)_{0} \omega, \omega\right) d \xi-\log \int_{\mathbb{S}^{n}} e^{n(\omega-\bar{\omega})} d \xi
$$

is $\geq 0$, with $=$ iff $e^{\omega}$ is a conformal factor.

The term conformal factor is again meant in the sense of (18). The analogue of the Möbius group, the conformal group of $\mathbf{S}^{n}$, is isomorphic to $\mathrm{SO}(n+1,1)$; the action of this group will be needed in more detail later. 
The operator $\left(P_{n}\right)_{0}$ is the GJMS operator evaluated at the round metric; the precise form of this operator was given in [7, Remark 2.23]. Namely, let

$$
\mathcal{B}=\sqrt{\Delta+\left(\frac{n-1}{2}\right)^{2}} .
$$

Then

$$
\left(P_{m}\right)_{0}=\prod_{a=1}^{m / 2} \underbrace{\left\{\left(\mathcal{B}+a-\frac{1}{2}\right)\left(\mathcal{B}-a+\frac{1}{2}\right)\right\}}_{\mathcal{B}^{2}-\left(a-\frac{1}{2}\right)^{2}} .
$$

Recall that the GJMS operator implicitly defined a local invariant via (19). Let

$$
\frac{n-m}{2} Q_{m}=P_{m} 1, \quad \text { so that } \quad P_{m}=P_{m}^{0}+\frac{n-m}{2} Q_{m},
$$

where $P_{m}^{0}=1$. In general, $P_{m}$ and $Q_{m}$ are not unique - for example, one may add any multiple of the norm-squared of the Weyl tensor, $|C|^{2}=C^{a b c d} C_{a b c d}$, to the Paneitz operator $P_{4}$ and still have a conformal covariant. What one really needs, for present purposes, is a choice of $P_{m}$ that is

- has principal part $\Delta^{m / 2}$;

- has conformal biweight $((n-m) / 2,(n+m) / 2)$;

- has coefficients which are rational in the dimension $n$;

- is formally self-adjoint; and

- annihilates the function 1 in dimension $n$.

Of course, such a $P_{m}$ is neither an operator on a particular manifold, nor an operator scheme for Riemannian manifolds in a certain dimension, but rather a formula, involving the dimension and some basis of the possible terms (built from $g, \nabla$, and $R$ ) which is stable for large dimensions.

There is certainly such a $P_{m}$ in the category of conformally flat metrics, and there is even an algorithm for writing it. Since such an operator must be $\Delta^{m / 2}$ for the flat metric $g_{a b}=\delta_{a b}$, we just need to write the operator invariantly for metrics $\left(g_{\omega}\right)_{a b}=e^{2 \omega} \delta_{a b}$. By the conformal covariance relation,

$$
\left(P_{m}\right)_{\omega}=e^{-(n+m) \omega / 2}\left(P_{m}\right)_{0} e^{(n-m) \omega / 2} .
$$

(As usual, we blur the distinction between a function $f$ and the operator $\mu_{f}$ of multiplication by $f$ - the final exponential in the foregoing formula should really be a multiplication operator.) In order to write this in terms of covariant derivatives in the metric $g_{\omega}$, we write $\left(P_{m}\right)_{0}$ as a composition of conformal covariants evaluated at the metric $g_{0}$, and use the conformal covariance relations for these "smaller" operators. For example,

$$
\left(P_{m}\right)_{0}=\Delta_{0}^{m / 2}=Y_{0}^{m / 2}=\left(e^{(n+2) \omega / 2} Y_{\omega} e^{-(n-2) \omega / 2}\right)^{m / 2},
$$

where $Y$ is the conformal Laplacian. As a result,

$$
\left(P_{m}\right)_{\omega}=e^{-(n+m) \omega / 2}\left(e^{(n+2) \omega / 2} Y_{\omega} e^{-(n-2) \omega / 2}\right)^{m / 2} e^{(n-m) \omega / 2} .
$$

Another way to compute would be to use the fact that $\Delta=\delta d$, and write

$$
\left(P_{m}\right)_{0}=\left(\delta_{0} d\right)^{m / 2}=\left(e^{n \omega} \delta_{\omega} e^{-(n-2) \omega} d\right)^{m / 2},
$$


using the metric invariance of $d$, and the conformal biweight $(n-2, n)$ of $\delta$. The result is

$$
\left(P_{m}\right)_{\omega}=e^{-(n+m) \omega / 2}\left(e^{n \omega} \delta_{\omega} e^{-(n-2) \omega} d\right)^{m / 2} e^{(n-m) \omega / 2} .
$$

At any rate, we now commute all covariant derivatives to the right, past the expressions in $\omega$. This produces terms in covariant derivatives of $\omega$. However, all second and higher derivatives of $\omega$ may be eliminated, in favor of local invariants, by iterated use of the Ricci tensor change formula

$$
\omega_{a b}=-V_{a b}-\omega_{a} \omega_{b}+\frac{1}{2} \omega_{c} \omega^{c} g_{a b}
$$

and its iterated covariant derivatives. (Here $\omega_{a \ldots b}$ abbreviates $\nabla_{b} \cdots \nabla_{a} \omega$.) The total degree of each term in $e^{\omega}$ is 0 , so we only have to worry about leftover occurrences of the first derivative, $\nabla \omega$. There are none such, however, as a consequence of our having picked the correct principal part and biweight for the conformal covariant $P_{m}$. The result is an expression for $P_{m}$ in the conformally flat category.

In the case of high-order bundle-valued conformal covariants, a procedure like that just above is also possible, given the correct principal part and biweight. (The correct principal part may be deduced from spectral considerations $[11,4]$.) In general, the principal part is written as a linear combination of compositions of "smaller" conformal covariants.

The subject of promoting conformal covariants in the conformally flat category to conformal covariants for general metrics still features some open questions. It is known [24] that the operator on scalar functions with principal part $\Delta^{3}$ does not promote in dimension 4 ; note that this is just beyond the restriction $m \geq n$ on the GJMS operators in even dimensions. In terms of Bernstein-Bernstein-Gelfand resolutions (see, e.g., [22]), which classify (differential) conformal covariants in the conformally flat category, one knows that operators which are not longest arrows do in fact promote. Some longest arrows (like the critical order GJMS operator $P_{n}$ ) promote as well. A plausible conjecture is that the $P_{n}$ are the only longest arrows that promote.

If we stay in the conformally flat category it is easily shown, by examining the above construction (which produces unique $P_{m}$ ), that the above conditions on $P_{m}$ hold. In addition [9], with the subscript 0 still denoting evaluation at the round sphere metric,

$$
\left(Q_{m}\right)_{0}=\frac{\Gamma\left(\frac{n+m}{2}\right)}{\Gamma\left(\frac{n-m+2}{2}\right)}, \quad \text { and in particular, } \quad\left(Q_{n}\right)_{0}=\Gamma(n) .
$$

This means that the first functional in $(21)$, which we have called $\mathcal{F}_{0}(\omega)$, is exactly the quantity estimated (i.e. asserted to be positive) by the exponential class inequality:

$$
\mathcal{F}_{0}(\omega):=\frac{n}{2 \Gamma(n)} \int_{\mathbb{S}^{n}}\left(P_{n} \omega, \omega\right) d \xi-\log \int_{\mathbb{S}^{n}} e^{n(\omega-\bar{\omega})} d \xi
$$

The issue is now whether we can get an estimate of the other term,

$$
\mathcal{F}(\omega):=\int_{\mathbb{S}^{n}}\left((B d \xi)_{\omega}-(B d \xi)_{0}\right),
$$

which is compatible with the exponential class estimate. This means that it should assert that

$$
C \mathcal{F}(\omega) \geq 0,
$$

where $C$ is the constant in (21) - the inequality should "go in the same direction as" the exponential class one. That is, it should assert this provided $C \neq 0$. If $C$ vanishes, then $\zeta_{A}(0)$ vanishes; one way to see this is that the log term comes from the volume penalty; the case when no penalty is needed is exactly $\zeta_{A}(0)=0$. It is not clear whether this can happen in reality; it might be reasonable to conjecture that $\zeta_{A}(0) \neq 0$ for all $A$ satisfying our assumptions. 
Remark 4. It would also be nice if our compatible inequalities were compatible in another way their cases of equality should contain the case of equality for the exponential class inequality. This will assure that the coupled functional vanishes exactly at conformal factors. But this additional property is provided automatically by the invariant nature of $\mathcal{F}(\omega)$ (Remark 3) the functional is diffeomorphism-invariant, and thus in particular is invariant under conformal diffeomorphisms. This guarantees that when $\omega$ is a conformal factor, $\mathcal{F}(\omega)$ will vanish.

To be more specific on how $P_{n}$ entered our formula for the $\mathcal{F}_{0}(\omega)$ part of the determinant quotient, recall first how the Gauss curvature prescription equation arose from the Yamabe equation. More generally, we would like a $Q_{n}$ prescription problem to arise in the same way from the $Q_{m}$ prescription problems, by dimensional regularization:

$$
\begin{gathered}
P_{m}=P_{m}^{0}+\frac{n-m}{2} Q_{m}, \quad P_{m}^{0} 1=0, \quad e^{\frac{n+m}{2} \omega}\left(P_{m}\right)_{\omega} f=\left(P_{m}\right)_{0}\left(e^{\frac{n-m}{2} \omega} f\right) \\
\begin{aligned}
\frac{n-m}{2}\left(Q_{m}\right)_{\omega} e^{\frac{n+m}{2} \omega} & =\left(P_{m}^{0}+\frac{n-m}{2} Q_{m}\right)_{0} e^{\frac{n-m}{2} \omega} \\
& =\left(P_{m}^{0}\right)_{0}\left(e^{\frac{n-m}{2} \omega}-1\right)+\frac{n-m}{2}\left(Q_{m}\right)_{0} e^{\frac{n-m}{2} \omega} .
\end{aligned}
\end{gathered}
$$

Dividing by $(n-m) / 2$ and evaluating at $n=m$, we have

$$
\left(Q_{n}\right)_{\omega} e^{n \omega}=\left(P_{n}^{0}\right)_{0} \omega+\left(Q_{m}\right)_{0}
$$

In particular, since $P_{n}^{0}$ has the form $\delta S d$ (by some invariant theory),

$$
\int\left(Q_{n} d v\right)_{\omega}=\int\left(Q_{n} d v\right)_{0}
$$

Going up an additional order in $\beta=(n-m) / 2$, as in the $m=2$ case, we get

$$
\left.\int \frac{\left(Q_{n} d v\right)_{\omega}-\left(Q_{n} d v\right)_{0}}{n^{\prime}-n}\right|_{n^{\prime}=n}=\frac{1}{2} \int \omega\left(P_{n}\right)_{0} \omega d v_{0}+\int \omega\left(Q_{n}\right)_{0} d v_{0} .
$$

In this way, $P_{n}$ has naturally entered the determinant calculation.

Here's the situation in dimension $n=4 . P_{4}$ is the Paneitz operator:

$$
P_{4}=\Delta^{2}+\delta T d+\frac{n-4}{2} Q_{4},
$$

where

$$
T=(n-2) J-4 V \cdot, \quad(V \cdot \varphi)_{b}=V_{b}^{a} \varphi_{a}, \quad Q_{4}=\frac{n}{2} J^{2}-2|V|^{2}+\Delta J .
$$

Since $\int U_{4}[A]$ is a conformal invariant, $U_{4}[A]$, which a priori is a linear combination of $K^{2}$, $|r|^{2},|R|^{2}$, and $\Delta K$ (or, equivalently, $J^{2},|V|^{2},|C|^{2}$, and $\Delta J$ ), must be something more special, namely a linear combination of $Q_{4}, \Delta J$, and $|C|^{2}(C=$ Weyl). Since these quantities have conformally invariant integrals, the leftover functional

$$
\int_{\mathbb{S}^{4}}\left((B d \xi)_{\omega}-(B d \xi)_{0}\right) d \xi
$$

can be expressed as a multiple of

$$
\int_{\mathbb{S}^{4}}\left(\left(J^{2} d \xi\right)_{\omega}-\left(J^{2} d \xi\right)_{0}\right) d \xi .
$$


$\left(J^{2}\right.$ could actually be replaced in this formula by anything linearly independent of $Q_{4}, \Delta J$, and $|C|^{2}$.)

In higher dimensions, because of the Conformal Index Theorem, there is always some leeway in the choice in $B$. By [14], if $L$ is a local invariant with $\int L$ conformally invariant, then in the conformally flat category,

$$
L=\text { const } \cdot \text { Pff }+\operatorname{div},
$$

where "div" is an exact divergence, and "Pff" is the Pfaffian (Euler characteristic density). Since $\int Q_{n}$ and $\int U_{n}$ are conformally invariant,

$$
U_{n}[A]=\text { const } \cdot Q_{n}+\operatorname{div},
$$

where the constant is uniquely determined by $A$.

In dimension 4 , the only possible universal divergence is $\Delta J$. Thus $J^{2}$ appears, basically, because it is a conformal primitive for $\Delta J$ :

$$
\left(\int J^{2}\right)^{\bullet}=2 \int J \Delta \omega=2 \int(\Delta J) \omega .
$$

As an overall conclusion in dimension 4, all of our penalized determinant quotient functionals end up being linear combinations of the two functionals

$$
\begin{aligned}
& \mathcal{F}_{0}(\omega)=\frac{1}{3} \int_{\mathbb{S}^{4}} \omega \Delta_{0}\left(\Delta_{0}+2\right) \omega d \xi-\log \int_{\mathbb{S}^{4}} e^{4(\omega-\bar{\omega})} d \xi, \\
& \mathcal{F}_{1}(\omega)=\int_{\mathbb{S}^{4}}\left\{\left(J^{2} d \xi\right)_{\omega}-\left(J^{2} d x\right)_{0}\right\} .
\end{aligned}
$$

By the above, we know all about the functional $\mathcal{F}_{0}(\omega)$. The other functional, $\mathcal{F}_{1}(\omega)$, is conveniently analyzed by the solution of the Yamabe problem $[40,36,1,34]$. The Yamabe quotient of the sphere $\mathbb{S}^{4}$ is

$$
\mathcal{Y}(f)=\frac{\int_{\mathbb{S}^{4}} f\left(\Delta_{0}+2\right) f d \xi}{\left(\int_{\mathbb{S}^{4}} f^{4} d \xi\right)^{1 / 2}} .
$$

The minimum value of $\mathcal{Y}(f)$ is attained at $f=1$, and so is 2 . Thus for nowhere-vanishing $f$,

$$
2\|f\|_{4}^{2} \leq \int_{\mathbb{S}^{4}} \underbrace{f\left(\Delta_{0}+2\right) f}_{f^{2} \cdot\left(\frac{\Delta_{0} f}{f}+2\right)} d \xi \leq \underbrace{\left\|f^{2}\right\|_{2}}_{\|f\|_{4}^{2}}\left\|\frac{\Delta_{0} f}{f}+2\right\|_{2},
$$

by the Schwarz inequality, so that

$$
2 \leq\left\|\frac{\Delta_{0} f}{f}+2\right\|_{2}
$$

We would like to apply this to the nowhere-vanishing function

$$
f=e^{\frac{n-2}{2} \omega}=e^{\omega} \text {. }
$$

This yields

$$
4 \leq \int_{\mathbb{S}^{4}}\left(e^{-\omega}(\underbrace{\Delta_{0}+2}_{Y_{0}}) e^{\omega}\right)^{2} d \xi=f_{\mathbb{S}^{4}} J_{\omega}^{2} d v_{\omega} .
$$

This result is well worth stating in its own right: 
Theorem 5. $0 \leq \int_{\mathbb{S}^{4}}\left(\left(J^{2} d \xi\right)_{\omega}-\left(J^{2} d x\right)_{0}\right)$, with $=$ iff $e^{\omega}$ is a conformal factor.

The last part, about the case of equality, comes from the case of equality for the Yamabe problem on $\mathbb{S}^{4}$. The inequality really expresses the instance $L_{1}^{2} \hookrightarrow L^{4}$ of the Sobolev Embedding Theorem in a way adapted to spectral invariant theory. As it has turned out, the functional $\mathcal{F}_{1}(\omega)$ has exactly the same case of equality as the exponential class functional $\mathcal{F}_{0}(\omega)$, not just a containing case of equality as described in Remark 4.

Just what are the coupling constants between the functionals $\mathcal{F}_{0}(\omega)$ and $\mathcal{F}_{1}(\omega)$ for some prominent admissible operators $A$ ? Up to overall constant factors, here are the functionals when $A$ is the conformal Laplacian, Dirac-squared, and Paneitz operators:

$$
\begin{array}{ll}
Y: & -3 \mathcal{F}_{0}(\omega)-2 \mathcal{F}_{1}(\omega), \\
\nabla^{2}: & 33 \mathcal{F}_{0}(\omega)+7 \mathcal{F}_{1}(\omega), \\
P_{4}: & 21 \mathcal{F}_{0}(\omega)-16 \mathcal{F}_{1}(\omega) .
\end{array}
$$

Note that by dimensional regularization, these numbers really fall out of formulas for $a_{4}[A]$, provided we keep enough information to read the dimension dependence. (See [10] for the $P_{4}$ calculation.)

Thus the extremal problem for $\mathcal{D}(Y)$ and $\mathcal{D}\left(\nabla^{2}\right)$ is solved, in the standard conformal class on $\mathbb{S}^{4}$. This calculation was originally done in [12], based on the determinant formula in [16].

For the penalized det $P_{4}$, functional, it's still unclear whether the round metrics are absolute extremals. The question can be phrased as comparing the sizes of the gaps (the differences between the left and right sides) in two sharp inequalities. The leading terms (those with the highest number of derivatives of $\omega$ ) for $\mathcal{F}_{0}+a \mathcal{F}_{1}$ produce a quadratic form that is

$$
\begin{array}{ll}
\text { positive definite for } & a \leq-8 / 15, \\
\text { indefinite for } & -8 / 15<a<-1 / 3, \\
\text { negative definite for } & a \geq-1 / 3 .
\end{array}
$$

The number for $P_{4}$ is $-16 / 21<-8 / 15$; this is suggestive of a minimum for $\mathcal{D}\left(\left(P_{4}\right)_{\omega}\right)$ at the round metrics, but the question is still open.

A look at dimension 6 indicates that something interesting is going on. The $\mathcal{D}$-functional in the standard conformal class on $\mathbb{S}^{6}$ is a linear combination of four functionals,

$$
\begin{aligned}
& \mathcal{F}_{0}(\omega)=\frac{1}{40} \int_{\mathbb{S}^{6}} \omega\left(\left(P_{6}\right)_{0} \omega\right) d \xi-\log \int_{\mathbb{S}^{6}} e^{6(\omega-\bar{\omega})} d \xi, \quad \mathcal{F}_{1}(\omega)=\int_{\mathbb{S}^{6}}\left(|d J|^{2} d \xi\right)_{\omega}, \\
& \mathcal{F}_{2}(\omega)=\int_{\mathbb{S}^{6}}\left(\left(|d J|^{2}+2 J^{3}\right) d \xi\right)_{\omega}-54, \\
& \mathcal{F}_{3}(\omega)=\int_{\mathbb{S}^{6}}\left(\left(|d J|^{2}+\frac{28}{5} J^{3}-\frac{48}{5} J|V|^{2}\right) d \xi\right)_{\omega}-108 .
\end{aligned}
$$

Each may be estimated to be nonnegative, with equality iff $e^{\omega}$ is a conformal factor. Thus we "win", for a given operator $A$, if there are no conflicting signs in the expression of the $\mathcal{D}$-functional for $A$ as

$$
a_{0} \mathcal{F}_{0}+a_{1} \mathcal{F}_{1}+a_{2} \mathcal{F}_{2}+a_{3} \mathcal{F}_{3}
$$

Remarkably, there are no conflicting signs in the cases of $Y$ and $\nabla^{2}$ : up to constant multiples, $\left(a_{0}, a_{1}, a_{2}, a_{3}\right)$ is

$$
\begin{aligned}
& Y: \quad(600,6,23,10), \\
& \nabla^{2}: \quad-(11460,93,556,365) .
\end{aligned}
$$

The nonnegativity of $\mathcal{F}_{1}(\omega)$ is clear; it vanishes iff $J$ is constant, iff (by Obata's Theorem) $\omega$ is a conformal factor. An analysis of $\mathcal{F}_{2}(\omega)$ may be done by considering the Yamabe quotient 
applied to the function $J$. The Yamabe quotient is

$$
\mathcal{Y}(u)=\frac{\int u(\Delta+2 J) u}{\|u\|_{3}^{2}}
$$

so the Yamabe constant (minimum of the Yamabe quotient) on $\mathbf{S}^{6}$ is 6 (setting $J=3$ and $u=1$ ). This gives

$$
\left.\int_{\mathbf{S}^{6}}\left(\left\{|d J|^{2}+2 J^{3}\right\}\right)_{\omega} \geq 6\left(\int_{\mathbf{S}^{6}}\left(|J|^{3} d \xi\right)_{\omega}\right)\right)^{2 / 3}
$$

since the Yamabe constant is conformally invariant (so in particular, it is the same at $g_{\omega}$ as at $g_{0}$ ). Now run an argument analogous to (24), based on (28) below, and replacing the Schwarz inequality step with an application of the Hölder inequality, with exponents $\frac{3}{2}$ and 3. (A very general version of this argument is given in [9, Corollary 3.6].) The result is the nonnegativity of $\mathcal{F}_{2}(\omega)$, with equality iff we are at a Yamabe metric, iff (by Obata's Theorem) $\omega$ is a conformal factor.

The estimate of $\mathcal{F}_{3}(\omega)$ is more subtle. By [39], there is a second-order conformal covariant $D$ on trace-free symmetric 2-tensors. One may compute [9] that for $n \geq 5$, this operator has positive definite leading symbol, is formally self-adjoint, and has has positive spectrum on the round sphere. Since the sign of the bottom eigenvalue (if any) of a conformal covariant is a conformally invariant quantity, $D$ has positive spectrum for metrics conformal to the round one. Thus for $n \geq 5$,

$$
\int_{\mathbf{S}^{n}}\left(D_{\omega} \varphi, \varphi\right)(d v)_{\omega} \geq 0
$$

with equality iff $\varphi=0$. If we substitute the trace-free Ricci tensor $b_{\omega}$ of the metric $g_{\omega}$ for $\varphi$, the result (when $n=6$ ) is the inequality $\mathcal{F}_{3}(\omega) \geq 0$. Furthermore, we have equality iff $b_{\omega}=0$, iff $\left(\mathbf{S}^{6}, g_{\omega}\right)$ is a space of constant curvature, iff $\omega$ is a conformal factor.

An interesting aspect of the determinant calculation for the conformal Laplacian in dimension 6 is the appearance of certain $n-8$ factors. In fact,

$$
\begin{aligned}
(4 \pi)^{n / 2} 7 ! a_{6}[Y]= & (n-8) \int\left\{-3(n-6)|d J|^{2}-\frac{1}{9}\left(35 n^{2}-266 n+456\right) J^{3}\right. \\
& \left.+\frac{2}{3}(n-1)(7 n-30) J|V|^{2}-\frac{2}{9}\left(5 n^{2}-2 n-48\right) \operatorname{tr} V^{3}\right\} d v \\
= & -\frac{5}{3}(n-8) \int Q_{6} d v+(n-8)(n-6) \int\left\{-\frac{13}{6}|d J|^{2}\right. \\
& \left.-\frac{1}{36}(125 n-314) J^{3}+\frac{2}{3}(7 n-5) J|V|^{2}-\frac{2}{9}(5 n+28) \operatorname{tr} V^{3}\right\} d v .
\end{aligned}
$$

This is analogous to an $n-6$ factor that appeared in the 4-dimensional case. The root cause of this phenomenon is the relative conformal invariance of the heat invariant $U_{n-2}[Y]$. To our knowledge, this was first remarked upon by Schimming [33], in connection with studies of Huygens' principle for hyperbolic equations. A Riemannian geometry proof is given in [30], based on the fact that $U_{n-2}[Y]$ is the coefficient of the "first log term" in the radial expansion of the fundamental solution (Green's function) of $Y$. [13] shows that this phenomenon is really a consequence of the conformal index property: take a fixed operator of the form $D=-\nabla^{a} \nabla_{a}-E$, and consider perturbations

$$
e^{-2 \varepsilon \omega}(D-\delta F)
$$


where $\varepsilon$ and $\delta$ are real parameters and $\omega$ and $F$ are functions. Now compute

$$
\left.\frac{\partial^{2}}{\partial \delta \partial \varepsilon}\right|_{(\delta, \varepsilon)=(0,0)}
$$

of the heat expansion with each of the two possible partial derivative orderings; the result is the relative conformal invariance of $U_{n-2}[Y]$ :

$$
U_{n-2}[Y]_{\omega}=e^{-(n-2) \omega} U_{n-2}[Y]_{0} .
$$

(That is, the conformal variation of $e^{(n-2) \omega} U_{n-2}[Y]_{\omega}$ vanishes.) In the conformally flat category, this forces $U_{n-2}[Y]$ to vanish. Thus, for example, $U_{6}[Y]$ is forced to have an overall factor of $n-8$.

This calculation gives no special conclusion for powers of a conformal covariant (like $\nabla^{2}$ ). Replacing $Y$ by a higher-order operator leads to the relative conformal invariance of a different term in the heat asymptotics; for example, $U_{n-4}[P]$ in the case of the Paneitz operator $P$.

Recently, Larry Peterson and the author [17] have computed the determinant quotient for the conformal Laplacian in the standard conformal class on $\mathbf{S}^{8}$. The starting point is a general formula for $U_{8}[D]$ due to Avramidi [2], where $D$ is any operator of Laplace type. We use this to compute $a_{8}[Y]$ in arbitrary dimension $n$, in the conformally flat category. The result is $(4 \pi)^{-n / 2}$ times a linear combination (with rational-in- $n$ coefficients) of the integrals of the nine scalar invariants

$$
(\Delta J)^{2}, \quad J|d J|^{2}, \quad J|\nabla V|^{2}, \quad(V, d J \otimes d J), \quad J^{4}, \quad J^{2}|V|^{2}, \quad J \operatorname{tr} V^{3}, \quad|V|^{4}, \quad \operatorname{tr} V^{4} .
$$

Here, for example, $\operatorname{tr} V^{3}=V^{a}{ }_{b} V_{c}^{b} V^{c}{ }_{a}$. Though this is a long calculation, something of a check is performed by the expected $n-10$ factor - the fact that this factor appears is reassuring. We have not yet been able to use this to solve the extremal problem for $\mathcal{D}\left(Y, g_{\omega}\right)$, but several sharp inequalities are available.

An interesting aspect of the extremal problem on low-dimensional spheres is the "checkerboard pattern"

\begin{tabular}{|c|c|c|}
\hline & $\operatorname{det} Y$ & $\operatorname{det} \not^{2}$ \\
\hline $\mathbf{S}^{2}$ & $\max$ & $\min$ \\
\hline $\mathbf{S}^{4}$ & $\min$ & $\max$ \\
\hline $\mathbf{S}^{6}$ & $\max$ & $\min$ \\
\hline
\end{tabular}

Here "max" means that the (suitably penalized) determinant quantity attains a max at the at the round metric and its conformal diffeomorphs.

In (as yet inconclusive) work with Carlo Morpurgo and Bent Ørsted, we have attempted to explain at least the $\max / \min$ alternation for the conformal Laplacian as follows. Suppose $n$ is even. Morpurgo shows that for real $s$ in the interval $\left(\frac{n}{2}-1, \frac{n}{2}\right)$, the zeta difference

$$
\zeta_{Y_{\omega}}(s)-\zeta_{Y_{0}}(s)
$$

is nonnegative. This difference has potential simple poles at the positive integers $\leq n / 2$, so if all these poles are realized, and no zeros intervene, we have a quantity of sign $(-1)^{\frac{n}{2}-1}$ for small positive $s$; by the conformal index property, this should also be the sign of the log-determinant difference

$$
\zeta_{Y_{\omega}}^{\prime}(0)-\zeta_{Y_{0}}^{\prime}(0)
$$

Recalling the minus sign in the definition of the determinant as $\exp \left(-\zeta^{\prime}(0)\right)$, this agrees with the $n=2,4,6$ cases in the above table. 
Considering what happens as we move along curves in the conformal class, by the obvious reflection principle, intervening zeros of (26) on the real axis must either come in pairs (i.e. be double zeros), or must move along the real axis. With double zeros, no harm is done to the above argument. A single zero, though, has the potential to reverse the sign of the above result, or ruin the result entirely, if it moves past $s=0$. As it moves through $s=0$, what we see is a zero of the log-determinant difference (27). So the problem is to rule these out - clearly a restatement of the assertion that the round metric is an absolute extremal. (Among the many things needed to make this discussion precise is to account for the conformal diffeomorphisms, which preserve the zeta function.)

In any case, what is missing is some sort of general-dimension approach to the convexity properties of the determinant quotient. This would avoid the dimension-by-dimension approach, and have the side benefit of proving sharp harmonic-analytic inequalities rather than just using them. This is conceptually related to the topic of complementary series in representation theory, as we shall try to explain in the next section.

\section{The complementary series}

The constants and differential operators that appear in the sharp inequalities that estimate quantities like those in (22) and (25), and in turn the determinant, also appear in the study of the complementary series of $\mathrm{SO}_{0}(n+1,1)$.

The conformal transformations $h$ of any Riemannian manifold, $\operatorname{ctran}(M, g)$, form a group. Recall that such a transformation has

$$
h \cdot g=\left(h^{-1}\right)^{*} g=\Omega_{h}^{2} g, \quad 0<\Omega_{h} \in C^{\infty}(M) .
$$

The corresponding infinitesimal notion is that of conformal vector fields $X$; these satisfy

$$
\mathcal{L}_{X} g=2 \omega_{X} g, \quad \omega_{X} \in C^{\infty}(M),
$$

where $\mathcal{L}$ is the Lie derivative, and form a Lie algebra $\operatorname{cvf}(M, g)$. The conformal factors satisfy cocycle conditions

$$
\Omega_{h_{1} \circ h_{2}}=\Omega_{h_{1}}\left(h_{1} \cdot \Omega_{h_{2}}\right), \quad \omega_{\left[X_{1}, X_{2}\right]}=X_{1} \omega_{X_{2}}-X_{2} \omega_{X_{1}} .
$$

The cocycle conditions are equivalent to the assertion that the family (series, in representation theoretic parlance) of maps

$$
u_{a}(h)=\Omega_{h}^{a} h \cdot, \quad U_{a}(X)=\mathcal{L}_{X}+a \omega_{X} \quad(a \in \mathbb{C})
$$

are homomorphisms into the group of automorphisms (resp. Lie algebra of endomorphisms) of functions on $M$, or in fact tensors, or tensor-spinors, of any type. The isometry subgroup (subalgebra) is defined by the condition $\Omega_{h}=1\left(\omega_{X}=0\right)$.

When $(M, g)$ is standard $\mathbf{S}^{n}$, these objects give the principal series of $\mathrm{SO}_{0}(n+1,1)$ (or $\operatorname{Spin}_{0}(n+1,1)$ if spinors are involved). That is,

$$
\mathbf{S}^{n}=G / M A N=K / M,
$$

where

$$
G=\mathrm{SO}_{0}(n+1,1), \quad M=\mathrm{SO}(n), \quad A \cong \mathbb{R}_{+}, \quad N \cong \mathbb{R}^{n}, \quad K=\mathrm{SO}(n+1) .
$$

The representations are $\operatorname{Ind}_{M A N}^{G} \lambda \otimes \nu \otimes 1$, with the interpretations

$$
\lambda \approx \text { tensor }- \text { spinor species; } \quad \nu \approx \text { conformal weight. }
$$


The stereographic map $\mathbb{R}^{n} \hookrightarrow \mathbb{S}^{n}$ is a special case of the more general Iwasawa decomposition theoretic map

$$
\bar{N} \hookrightarrow G=\bar{N} M A N \rightarrow G / M A N .
$$

Such induced representations are best thought of algebraically at first, as $(\mathfrak{g}, K)$-modules. This means: take the $K$-finite vectors, so one has a direct sum of $K$-modules. The action of $\mathfrak{g}$ lets you travel among these modules - you can get from a $K$-module $\alpha$ to a $K$-module $\beta$ only if $\mathfrak{s} \otimes \alpha$ has a $\beta$ summand in its $K$-decomposition (the selection rule). Here

$$
\mathfrak{g}=\mathfrak{k}+\mathfrak{s}
$$

is the Cartan decomposition.

An example is the expansion into spherical harmonics of functions on $\mathbb{S}^{n}$ :

$$
\mathcal{E} \cong{ }_{\mathrm{SO}(n)} E_{j} .
$$

Using the big Lie algebra $\mathfrak{s o}(n+1,1)$, we can move up and down the ladder,

$$
E_{j-1} \leftarrow E_{j} \rightarrow E_{j+1},
$$

within the spherical principal series representations

$$
\operatorname{Ind}_{M A N}^{G} 1 \otimes \nu \otimes 1=:\left(U_{\frac{n}{2}+\nu}, u\right) .
$$

The unitaries in this series are at imaginary $\nu$ (the unitary principal series), and, on the real axis, in the interval $|\nu|<n / 2$ - the complementary series. The inner products on the complementary series representations $\left(U_{\frac{n}{2}+\nu}, u\right)$ have the form

$$
(\varphi, \psi)_{\nu}=\int_{\mathbb{S}^{n}} \varphi\left(A_{2 \nu} \bar{\psi}\right) d \xi
$$

where $A_{2 \nu}$ is the intertwining operator

$$
A_{2 \nu}:\left(U_{\frac{n}{2}+\nu}, u\right) \rightarrow\left(U_{\frac{n}{2}-\nu}, u\right) .
$$

Since the two representations $U_{\frac{n}{2} \pm \nu}$ are naturally dual (they live in section spaces of the bundles of $\mp \nu$-densities), the integrand is natural.

The Knapp-Stein intertwinor [26] is an integral operator, the integral converging in a certain range of $\operatorname{Re}(\nu)$. Differential intertwinors, like the realization of the conformal Laplacian and Paneitz operator on the sphere, live in the analytic continuation (in $\nu$ ) of the family of KnappStein intertwinors. In the sense of pseudo-differential operators and of complex powers of an elliptic operator [35], assuming this analytic continuation,

$$
A_{2 \nu}=\Delta^{\nu}+\text { (lower order). }
$$

Up to a constant, $\left(P_{m}\right)_{0}$ is $A_{m}$. Not surprisingly, the obvious continuation of the formula (23) given earlier for $\left(P_{m}\right)_{0}$ turns out to be a formula for $A_{2 \nu}($ all $\nu)$ :

$$
A_{2 \nu}=\frac{\Gamma\left(\mathcal{B}+\nu+\frac{1}{2}\right)}{\Gamma\left(\mathcal{B}-\nu+\frac{1}{2}\right)},
$$

where (recall)

$$
\mathcal{B}=\sqrt{\Delta+\left(\frac{n-1}{2}\right)^{2}} .
$$


For $|\nu|<n / 2$, by Stirling's formula for the Gamma function, the complementary series norm on $U_{\frac{n}{2}+\nu}$ is a Sobolev $L_{\nu}^{2}$ norm.

The fact that the complementary series norm is an invariant Sobolev norm follows from the intertwining property:

$$
\begin{gathered}
\int_{\mathbb{S}^{n}} \varphi \cdot A_{2 \nu}\left\{\left(\mathcal{L}_{X}+\left(\frac{n}{2}-\nu\right) \omega_{X}\right) \psi\right\} d \xi=\int_{\mathbb{S}^{n}} \varphi \cdot\left(\mathcal{L}_{X}+\left(\frac{n}{2}+\nu\right) \omega_{X}\right) A_{2 \nu} \psi d \xi \\
=\int_{\mathbb{S}^{n}}\left\{\left(-\mathcal{L}_{X}-\left(\frac{n}{2}-\nu\right) \omega_{X}\right) \varphi\right\} \cdot A_{2 \nu} \psi d \xi .
\end{gathered}
$$

Here we're using the fact that the case $\nu=0$ is in the unitary principal series; this really follows from the simpler fact that the volume form $E$ has

$$
\mathcal{L}_{X} E=n \omega_{X} E .
$$

This last formula may be viewed as the origin of the rho-shift $n / 2$. This also happens more generally in the theory of parabolic induction for semisimple Lie groups; the rho-shift due to the $(\mathfrak{g}, \mathfrak{a})$ root system is related to the response of the natural volume form on $G / M A N$ to infinitesimal transformations from $\mathfrak{g}$. In the present case, the calculation is

$$
\begin{aligned}
0= & \int \underbrace{\mathcal{L}_{X}}_{d \iota(X)}\left(\varphi \cdot A_{2 \nu} \psi d \xi\right)=\int \varphi\{\underbrace{\left(\mathcal{L}_{X}+\left(\frac{n}{2}+\nu\right) \omega_{X}\right) A_{2 \nu}}_{A_{2 \nu}\left(\mathcal{L}_{X}+\left(\frac{n}{2}-\nu\right) \omega_{X}\right)} \psi\} d \xi \\
& +\int\left\{\left(\mathcal{L}_{X}-\left(\frac{n}{2}+\nu\right) \omega_{X}\right) \varphi\right\} A_{2 \nu} \psi \cdot d \xi+\int \varphi\left(A_{2 \nu} \psi\right) n \omega_{X} d \xi \\
= & \int \varphi\left\{A_{2 \nu}\left(\mathcal{L}_{X}+\left(\frac{n}{2}-\nu\right) \omega_{X}\right) \psi\right\} d \xi+\int\left\{\left(\mathcal{L}_{X}+\left(\frac{n}{2}-\nu\right) \omega_{X}\right) \varphi\right\} A_{2 \nu} \psi \cdot d \xi .
\end{aligned}
$$

There's another natural invariant norm on the space carrying $U_{\frac{n}{2}-\nu}$, namely the $L^{p}$ norm with

$$
p=\frac{2 n}{n+2 \nu} .
$$

Note that

$$
p \in(1, \infty) \Longleftrightarrow \nu \in\left(-\frac{n}{2}, \frac{n}{2}\right) .
$$

The invariance calculation is

$$
0=\int \mathcal{L}_{X}\left(\varphi^{p} d \xi\right)=p \int \varphi^{p-1}\left(\mathcal{L}_{X} \varphi\right) d \xi+\int \varphi^{p} n \omega_{X} d \xi=p \int \varphi^{p-1}\left(\mathcal{L}_{X}+\frac{n}{p} \omega_{X}\right) f d \xi
$$

this shows that when

$$
\frac{n}{p}=\frac{n}{2}+\nu,
$$

we have an invariant norm:

$$
\int \varphi^{p} d \xi=\int h \cdot\left(\varphi^{p} d \xi\right)=\int(h \cdot \varphi)^{p} \underbrace{h \cdot d \xi}_{\Omega_{h}^{n} d \xi}=\int \Omega_{h}^{-p\left(\frac{n}{2}+\nu\right)}\left(u_{\nu}(h) \varphi\right)^{p} \Omega_{h}^{n} d \xi .
$$

The Sobolev embedding inequalities

$$
L_{\nu}^{2} \hookrightarrow L^{\frac{2 n}{n-2 \nu}}, \quad L^{\frac{2 n}{n+2 \nu}} \hookrightarrow L_{-\nu}^{2} \quad(\nu \geq 0),
$$


in the sharp form due to Beckner [5], compare these two invariant norms for $\nu$ between $-n / 2$ and 0 , saying that saying that

$$
\max _{L^{p} \backslash 0} \frac{\left|(\varphi, \psi)_{\nu}\right|}{\|\varphi\|_{p}\|\psi\|_{p}}
$$

is attained exactly at conformal factors: both $\varphi$ and $\psi$ should be constant multiples of $\Omega_{h}^{\frac{n}{2}-\nu}$, where $h$ is a conformal transformation with $\left(h^{-1}\right)^{*} g=\Omega_{h}^{2} g$. This gives a comparison of norms, and of norms on the corresponding dual spaces. These lead, by endpoint differentiation, to the exponential class inequality (22). They are also used in deriving geometric inequalities like the estimate on $\mathcal{F}_{2}(\omega)$ in (25), based on [9, Corollary 3.6].

This interpretation in terms of representation theory is very clean and nice, but unfortunately, it has never been put to work in actually proving sharp inequalities. This remains a prospect for the future, much like (and probably closely related to) that of proving convexity properties of the determinant, and using them to solve extremal problems. The $L^{p}$ norms in (28) are quite amenable to hard geometro-analytic methods like symmetric decreasing rearrangement, while representation theory is still quite linear (meaning quadratic in the present formulation - that is, concerned with inner products).

\section{References}

[1] Aubin T., Équations differentielles non linéares et problème de Yamabe concernant la courbure scalaire, J. Math. Pures Appl. (9) 55 (1976), 269-296.

[2] Avramidi I.G., A covariant technique for the calculation of the one-loop effective action, Nuclear Phys. B 355 (1991), 712-754, Errata, Nuclear Phys. B 509 (1998), 557-558.

[3] Avramidi I.G., Branson T., Heat kernel asymptotics of operators with non-Laplace principal part, Rev. Math. Phys. 13 (2001), 847-890, math-ph/9905001.

[4] Avramidi I.G., Branson T., A discrete leading symbol and spectral asymptotics for natural differential operators, J. Funct. Anal. 190 (2002), 292-337, hep-th/0109181.

[5] Beckner W., Sharp Sobolev inequalities on the sphere and the Moser-Trudinger inequality, Ann. of Math. (2) 138 (1993), 213-242.

[6] Branson T., Differential operators canonically associated to a conformal structure, Math. Scand. 57 (1985), 293-345.

[7] Branson T., Group representations arising from Lorentz conformal geometry, J. Funct. Anal. 74 (1987), 199-291.

[8] Branson T., The functional determinant, Lecture Note Series, Vol. 4, Global Analysis Research Center, Seoul National University, 1993.

[9] Branson T., Sharp inequalities, the functional determinant, and the complementary series, Trans. Amer. Math. Soc. 347 (1995), 3671-3742.

[10] Branson T., An anomaly associated to 4-dimensional quantum gravity, Comm. Math. Phys. 178 (1996), 301-309.

[11] Branson T., Stein-Weiss operators and ellipticity, J. Funct. Anal. 151 (1997), 334-383.

[12] Branson T., Chang S.-Y.A., Yang P., Estimates and extremals for zeta function determinants on fourmanifolds, Comm. Math. Phys. 149 (1992), 241-262.

[13] Branson T., Gilkey P., The asymptotics of the Laplacian on a manifold with boundary, Comm. Partial Differential Equations 15 (1990), 245-272.

[14] Branson T., Gilkey P., Pohjanpelto J., Invariants of conformally flat manifolds, Trans. Amer. Math. Soc. 347 (1995), 939-954.

[15] Branson T., Ørsted B., Conformal indices of Riemannian manifolds, Compos. Math. 60 (1986), $261-293$.

[16] Branson T., Ørsted B., Explicit functional determinants in four dimensions, Proc. Amer. Math. Soc. 113 (1991), 669-682. 
[17] Branson T., Peterson L.J., in preparation.

[18] Browder F.E., Families of linear operators depending on a parameter, Amer. J. Math. 87 (1965), $752-758$.

[19] Carlen E., Loss M., Competing symmetries, the logarithmic HLS inequality and Onofri's inequality on $\mathbf{S}^{n}$, Geom. Funct. Anal. 2 (1992), 90-104.

[20] Dowker J.S., Kennedy G., Finite temperature and boundary effects in static space-times, J. Phys. A: Math. Gen. 11 (1978), 895-920.

[21] Eastwood M., Singer M., A conformally invariant Maxwell gauge, Phys. Lett. A 107 (1985), 73-74.

[22] Eastwood M., Slovák J., Semiholonomic Verma modules, J. Algebra 197 (1997), 424-448.

[23] Gilkey P., Invariance theory, the heat equation, and the Atiyah-Singer index theorem, CRC Press, Boca Raton, 1995.

[24] Graham C.R., Conformally invariant powers of the Laplacian, II. Nonexistence, J. London Math. Soc. (2) 46 (1992), 566-576.

[25] Graham C.R., Jenne R., Mason L., Sparling G., Conformally invariant powers of the Laplacian, I. Existence, J. London Math. Soc. (2) 46 (1992), 557-565.

[26] Knapp A., Stein E., Intertwining operators for semisimple Lie groups. II, Invent. Math. 60 (1980), 9-84.

[27] Onofri E., On the positivity of the effective action in a theory of random surfaces, Comm. Math. Phys. 86 (1982), 321-326.

[28] Palais R.S., Foundations of global non-linear analysis, Benjamin \& Co., New York, 1968.

[29] Paneitz S., A quartic conformally covariant differential operator for arbitrary pseudo-Riemannian manifolds, Preprint, 1983.

[30] Parker T., Rosenberg S., Invariants of conformal Laplacians, J. Differential Geom. 25 (1987), $199-222$.

[31] Penrose R., Rindler W., Spinors and space-time, Vol. I, Cambridge University Press, 1984.

[32] Riegert R., A non-local action for the trace anomaly, Phys. Lett. B 134 (1984), 56-60.

[33] Schimming R., Lineare Differentialoperatoren zweiter Ordnung mit metrischem Hauptteil und die Methode der Koinzidenzwerte in der Riemannschen Geometrie, Beitr. z. Analysis 15 (1981), 77-91.

[34] Schoen R., Conformal deformation of a Riemannian metric to constant scalar curvature, J. Differential Geom. 20 (1984), 479-495.

[35] Seeley R., Complex powers of an elliptic operator, Proc. Symposia Pure Math. 10 (1967), $288-307$.

[36] Trudinger N., Remarks concerning the conformal deformation of Riemannian structures on compact manifolds, Ann. Scuola Norm. Sup. Pisa (3) 3 (1968), 265-274.

[37] Weyl H., The classical groups: their invariants and representations, Princeton University Press, 1939.

[38] Widom H., Szegö's theorem and a complete symbolic calculus for pseudodifferential operators, in Seminar on Singularities, Editor L. Hörmander, Ann. of Math. Stud. 91 (1979), 261-283.

[39] Wünsch V., On conformally invariant differential operators, Math. Nachr. 129 (1986), 269-281.

[40] Yamabe H., On a deformation of Riemannian structures on compact manifolds, Osaka J. Math. 12 (1960), 21-37. 